\title{
Formation of colloidal threads in geometrically varying flow-focusing channels
}

\author{
V. Krishne Gowda, ${ }^{1, *}$ Cecilia Rydefalk $\odot,{ }^{1}$ L. Daniel Söderberg, ${ }^{2,3}$ and Fredrik Lundell ${ }^{1,3}$ \\ ${ }^{1}$ FLOW, Department of Engineering Mechanics, KTH Royal Institute of Technology, \\ SE-100 44 Stockholm, Sweden \\ ${ }^{2}$ Department of Fibre and Polymer Technology, KTH Royal Institute of Technology, \\ SE-100 44 Stockholm, Sweden \\ ${ }^{3}$ Wallenberg Wood Science Center, KTH Royal Institute of Technology, SE-100 44 Stockholm, Sweden
}

(Received 8 March 2021; accepted 5 October 2021; published 2 November 2021)

Threads of colloidal dispersions can be formed in microfluidic channel systems and are often used for analytical purposes or to assemble macroscopic structures from colloidal particles. Here, we report a combined experimental and numerical study of thread formation in channel systems with varying geometry. In the reference flow-focusing configuration, the sheath flows impinge the core flow orthogonally while in four other channel configurations, the sheath flows impinge the core flow at different confluence angles, which are both positive and negative with respect to the reference sheath direction. Tomographic measurements of the thread development are made using optical coherence tomography (OCT) and are compared to numerically simulated 3D data. The numerical simulations performed with an immiscible fluid solver show good agreement with the experiments in terms of 3D thread shapes, wetted region morphologies, and velocity fields provided an ultralow interfacial tension is applied between the low viscosity (solvent) sheath flows and the high viscosity (dispersion) core flow. Such an ultralow interfacial tension is motivated by the so-called Korteweg stresses induced as a result of the concentration gradient between two miscible fluids in nonequilibrium state. These stresses mimic the effect of interfacial tension, and are often modeled as an effective interfacial tension (EIT), an approach chosen in the present work as well. The value of interfacial tension applied in the simulations was determined through an optimization procedure and compares well with a value deduced from a scaling analysis utilizing the downstream development of experimentally determined thread shape. The experimental and numerical results show that for channel configurations with modest deviations from orthogonal sheath flows, the effect on the thread is similar regardless of whether the sheath flows are co- or counterflowing the core flow. In fact, for these cases, the effect of co- and counterflowing sheath flows can be reproduced with orthogonal sheath flows, if the sheath channel width is increased. However, for channel configurations with larger deviations from orthogonal sheath flows, the effects on the thread are direction dependent. The one-to-one comparison and analysis of numerical and experimental results bring useful insights to understand the behavior of miscible systems involving high-viscosity contrast fluids. These key results provide the foundation to tune the

\footnotetext{
*krivi@mech.kth.se
}

Published by the American Physical Society under the terms of the Creative Commons Attribution 4.0 International license. Further distribution of this work must maintain attribution to the author(s) and the published article's title, journal citation, and DOI. Funded by Bibsam. 
flow-focusing for specific applications, for example in tailoring the assembly of nanostructured materials.

DOI: 10.1103/PhysRevFluids.6.114001

\section{INTRODUCTION}

A boundary between two fluids (here, the term fluid is used in a generic form, encompassing both molecular liquids and suspensions) is identifiable, irrespective of whether the fluids are immiscible or miscible. In the case of immiscible fluids, the boundary zone is clearly distinguished through a distinct interface created by the equilibrium interfacial tension acting between the two fluids. In the context of miscible fluids, no such distinct interface exists as the two fluids are fully mixed at equilibrium. However, when two miscible fluids come into contact, a boundary between them can persist till an equilibrated homogeneous mixture have been formed due to diffusive mixing. This boundary zone can act as a de facto interface with some of its properties resembling that of a distinct interface observed between immiscible fluids [1-5]. In 1901, Korteweg first proposed that when two nonpremixed miscible fluids are brought into contact, the composition inhomogeneties and gradients of the fluid property at the zone of contact gives rise to additional stresses (so-called Korteweg stresses). These stresses effectively mimic capillarylike stress effects [6] across the boundary zone that can be seen as a sharp de facto interface. Accordingly, analogous to the interfacial tension $\gamma$ in the immiscible fluids, an effective interfacial tension (EIT) for miscible fluids in nonequilibrium state, commonly denoted as $\Gamma_{e}[7,8]$ can be written as

$$
\Gamma_{e}=K \frac{\Delta \Phi^{2}}{\delta}
$$

where $K$ is the Korteweg factor accounting for the relevant interaction effects (e.g., particle-solvent interactions in miscible complex fluids) between the two fluids, $\delta$ is the interface thickness and $\Delta \Phi$ is the variation in composition or volume fraction $\Phi$. As can be seen from Eq. (1), $\Gamma_{e}$ exists as long as the composition gradients persist at the de facto interface, and as the interface smears out due to diffusion over time, the EIT goes to zero.

Following Korteweg's work, numerous experimental [9-11] and numerical investigations [12-14] in the extant literature have invoked the phenomenon of EIT to rationalize the behavior of multi-fluid dynamical processes occurring at short times in nonequilibrium miscible systems. A few examples are microgravity experiments [15], evolution of miscible droplets [16,17], modeling of hydrodynamic instabilities like viscous fingering or in Hele-Shaw flows [18,19], stabilization of Rayleigh-Taylor instabilities induced by evaporation between a polymer solution and its own solvent [20] and so on. Some of the recent experimental techniques explored to measure the EIT between miscible fluids are through the evolution of drop shape [21,22], examination of hydrodynamic instabilities [7,23], and probing of capillary waves by light scattering [24,25]. In spite of these attempts, measuring the EIT between miscible fluids is intrinsically difficult due to ultralow values $\left(\Gamma_{e} \sim 10^{-4}-10^{1} \mathrm{mN} \mathrm{m}^{-1}\right)$ and absence of a distinct interface $[7,8,26]$.

For miscible systems, the Péclet number $\mathrm{Pe}=U h / D$ is the relevant nondimensional quantity to measure the relative importance of convective and diffusive effects, with $U$ being the flow velocity, $h$ the characteristic length, and $D$ the diffusion coefficient between the two fluids [5,27]. When a pair of miscible fluids is passed through a flow-focusing channel, the Péclet number dictates the flow-patterns. Flow-focusing essentially comprises a core fluid in the central channel focused by the sheath fluid $[28,29]$ entering from the side-channels as shown schematically in Fig. 1(a). At high Péclet number under laminar flow conditions, where diffusion is almost negligible, viscous threads are formed, while at low Péclet number, the threads undergo diffusive instabilities leading to a wide diversity of flow-patterns $[28,30,31]$. It is even observed that the miscible threads at high Péclet 


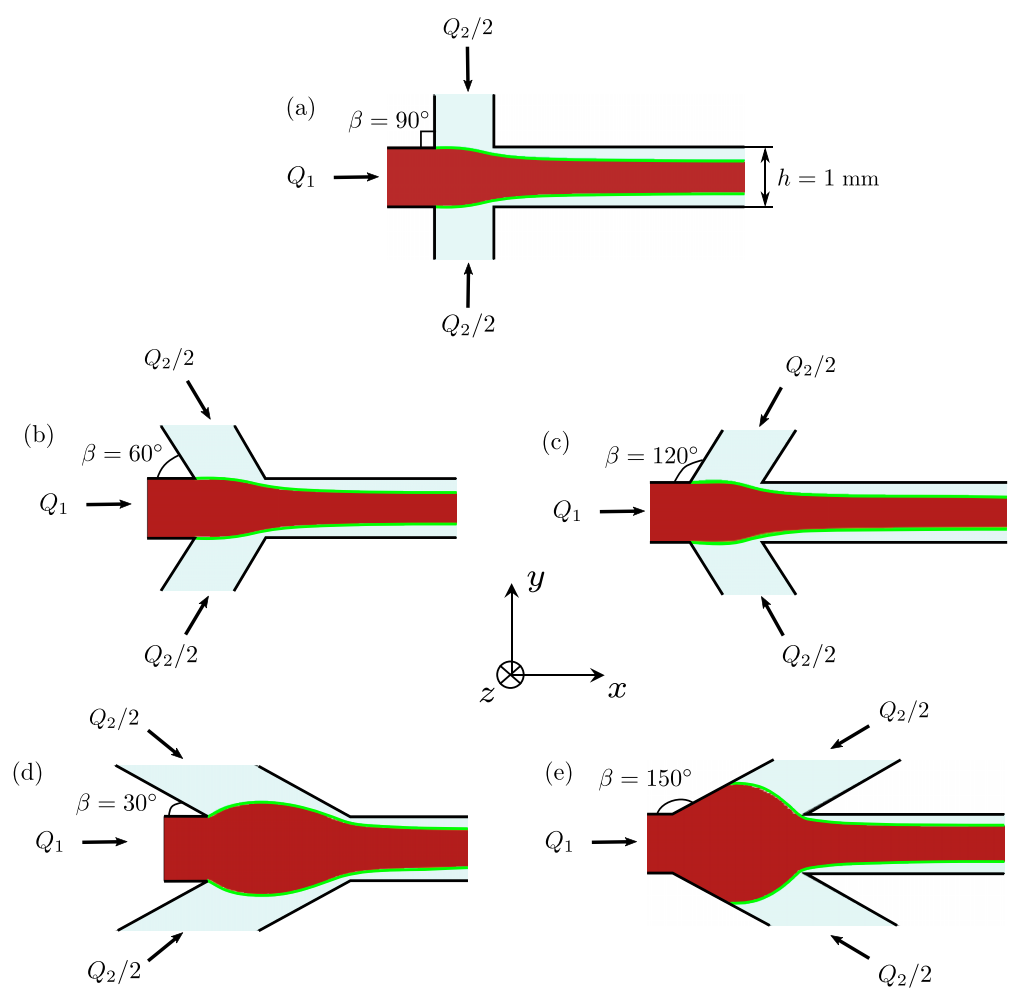

FIG. 1. (a)-(e) Schematic illustration of the top view of geometrically varying flow-focusing channel configurations with various confluence angles $\beta$. The red color represents the core fluid entering the central inlet channel with a volumetric flow rate $Q_{1}$. The light cyan color denotes the sheath fluid entering from the side inlet channels at an angle $\beta$ with a flow rate of $Q_{2} / 2$ in each channel. The central channel has a square cross-section with sidelength $h$.

number is equivalent to the multi-fluid viscous flow problem, and could be employed to examine and realize the role of diffusion [29,32].

For a high Péclet number system, with a colloidal dispersion as the core fluid and its own solvent as the sheath fluid, we have detected and reported that the characteristics of spatial thread evolution could be accurately captured and modeled as an immiscible fluid problem with a very weak interfacial tension $\gamma$ for a set of flow rates and specified rheology of the two fluids [33]. Ideally, at high Péclet number, the timescale for interdiffusion between the colloidal dispersion and its own solvent is almost negligible compared to the convective timescale of the two fluids in the channel. In such a scenario, presence of a sharp de facto interface due to composition gradients is expected in the experiments $[5,7,8]$. However, it is extremely difficult to access such a sharp de facto interface between the two fluids experimentally. The miscible viscous thread structures formed at high Péclet number are in an out-of-equilibrium state and occur before the two fluids are fully mixed.

In such occurrences, it is possible to reckon the de facto interface by applying a weak interfacial tension $\gamma$ in numerical modeling. In reality, the weak interfacial tension $\gamma$ accounts for the Korteweg stresses induced by the composition gradients in the experiments. In the previous work [33], the experimental observations were reproduced numerically with a very weak interfacial tension $\gamma$ $\left(\mathcal{O} \sim 10^{-2} \mathrm{mN} \mathrm{m}^{-1}\right)$. Moreover, the magnitude of $\gamma$ corroborated with the experimental measurements of previous studies conducted for miscible complex fluids involving colloidal dispersions or 
polymer solutions and its own solvent $[8,26]$. Thus, it was established that the very weak interfacial tension $\gamma$ acts as an effective interfacial tension in the experiments with $\gamma \equiv \Gamma_{e}$.

Furthermore, for a finite length of the channel, the estimation of time and length scales derived from a scaling model [33], showed the streamwise spatial evolution of the high-viscosity thread from near-ellipsoidal cross-section to circular shape was found to be dependent on ultralow interfacial tension. Similar to the capillary number in immiscible systems, an effective capillary number based on $\Gamma_{e}$ for the nonequilibirum miscible system could be defined as

$$
\mathrm{Ca}_{e}=\frac{\eta Q}{h^{2} \Gamma_{e}},
$$

where $\eta$ is the dynamic viscosity, $Q$ is the flow rate of the core fluid thread, and $h$ is the width of channel. Thus, when the streamwise spatial coordinate is normalised with $\mathrm{Ca}_{e}$, the spatial evolution of the thread heights at different $\gamma\left(\Gamma_{e}\right)$ collapse on to a master curve. In other words, in the framework of Korteweg's theory for miscible fluids, employing a microfluidic flow-focusing setup, we have proposed a methodology with a possibility to determine the EIT between two miscible fluids. In short, $\Gamma_{e}$ can be estimated by measuring the spatial evolution of the thread shape formed by these two fluids, and equating it with the master curve. However, this method was not yet fully exploited.

In the present work, we widen the studies of viscous thread formation at high Péclet number in miscible environments into a systematic investigation employing geometrically varying flowfocusing setups with a confluence angle $\beta$ as illustrated in Fig. 1. The Confluence angle $\beta$ refers to the angle made by the side (sheath) flow channel inlets with the central (core) flow channel inlet. We characterise the flow in three dimensions (3D) both experimentally and numerically employing optical coherence tomography (OCT) and volume of fluid (VoF) method [34] implemented in the open-source code OpenFOAM [35].

There are three profound objectives for this study. The first is to investigate the effect of confluence angles on the 3D shape of the thread structures. Here, we aim to understand the influence of sheath fluid impingement and geometrical channel effects on the thread formation and wetted region morphologies. Through this, we address an important aspect: whether the process of thread detachment from the top and bottom walls of the channel in miscible systems [28,32] occurs (i) naturally through the self-lubrication principle [30], a phenomenon associated with the effect of viscous dissipation of energy and originally observed in core-annular flow involving high-viscosity contrast immiscible fluid pairs [3,36], or (ii) through some other mechanisms with respect to highviscosity contrast miscible fluid pairs. The effects of confluence angle $\beta$ could also be potentially useful to understand how the sheath flow momentum affects the system, and identify the means to achieve efficient extensional flows. The second objective is, to exploit the experimentally measured $3 \mathrm{D}$ spatial evolution of the thread shape together with an effective capillary number dependent master curve to estimate an experimentally intractable variable, namely the $\Gamma_{e}$ between two miscible fluids. The final goal is to bring out the implications of ultralow interfacial tension in general and EIT in particular in microfluidic flow systems. In addition to these, a valuable feature of microfluidic flows is demonstrated, where the 3D flow characteristics of different confluence angle geometries can be replicated with a geometry of $\beta=90^{\circ}$ by altering the sheath flow channel widths. The present study also illustrates how additional insights into the physical mechanisms acting in the complex microfluidic systems can be gained from a diligent analysis of numerical calculations. These insights are difficult to ascertain in experiments due to inherent composition-dependent fluid properties.

As the experimental fluids, similar to our previous work [33], we use a colloidal dispersion for the core and its own solvent as the sheath fluid. The ingredients of the colloidal dispersion consists of cellulose nanofibrils (CNF) dispersed in water. Such nanofibrils have been assembled into highperformance structural cellulose filaments via hydrodynamic focusing [37,38]. Understanding the underlying flow behavior among various geometrical configurations is critical for controlling the 
hydrodynamic assembly [39,40]. Moreover, these colloidal systems exhibit variant compositiondependent fluid properties based on particle structure and interparticle interactions, and the $\Gamma_{e}$ for such colloidal dispersion and its solvent system is expected to vary considerably $[8,41]$.

The organization of the paper is as follows. In Sec. II, we give an overview of the experimental setup and a brief outline of the numerical method. In Sec. III A, we compare and discuss the numerical and experimental 3D thread topologies and wetted region morphologies on the top and bottom walls for various flow-focusing configurations emphasizing the role of confluence angle $\beta$. In Sec. III B, we briefly recall the scaling model, and the master curve, and use it to estimate the $\Gamma_{e}$ for the present experimental fluids. The estimated $\Gamma_{e}$, in turn, is verified with the value of $\Gamma_{e}$ obtained through an optimization procedure. In Sec. III C, numerical and experimental results of the centreline velocity is compared. Further, a numerical comparison of strain rates along the centreline in different geometrical configurations is undertaken to dissect the effects of confluence angle $\beta$ on the flow-field. In Sec. IV, replicability of the 3D flow features of different confluence angles $\beta$ in modified flow-focusing channels with $\beta=90^{\circ}$ is presented. In Sec. V, a remark highlighting the significance of EIT in microfluidic channels is elucidated, and finally a brief summary of the conclusions is provided in Sec. VI.

\section{EXPERIMENTAL AND NUMERICAL SETUPS}

\section{A. Experimental setup}

\section{Flow-focusing geometries}

In this work, we employ five distinct types of flow-focusing channel setups. The setups are planar with square cross-sections of side-length $h=1 \mathrm{~mm}$ and have the geometrical configurations as shown in Fig. 1. All the geometries have one main central inlet channel for the core flow and two sheath flow inlets inclined with a confluence angle $\beta$. The confluence angle is varied between $30^{\circ}$ and $150^{\circ}$ with an interval of $30^{\circ}$, to systematically investigate the impact of sheath flow impingement and channel effect on the core fluid 3D thread topology and flow-field.

All the channel geometries are built using a stainless-steel plate of $1 \mathrm{~mm}$ thickness similar to our previous works involving flow-focusing configuration with confluence angle $\beta=$ $90^{\circ}[33,37,39,40]$. The steel channel plate is enclosed on both sides with layers of aluminum plates and a cyclic olefin copolymer (COC) film forming an assembly of "aluminum plate-COC-steel channel-COC-aluminum plate" sandwich. The fluids are injected at constant volumetric flow rates into the core and sheath flow channel inlets by two syringe pumps (WPI, AI-4000). Furthermore, all the geometrical configurations [Figs. 1(b), 1(c) and 1(d), 1(e)] will be discussed in relative to the reference configuration [Fig. 1(a)], and this choice will be clarified in Sec. III A.

In all the configurations, the experimental measurements are performed at constant volumetric flow rates (see Fig. 1) with $Q_{1}=6.5 \mathrm{~mm}^{3} \mathrm{~s}^{-1}$ and $Q_{2}=7.5 \mathrm{~mm}^{3} \mathrm{~s}^{-1}$, respectively. The core fluid is a colloidal dispersion exhibiting a non-Newtonian viscosity behavior as shown in Fig. 2. The sheath fluid is a deionized (DI) water of viscosity $\eta_{2}=1 \mathrm{mPa} \mathrm{s}$.

\section{Dispersion material and its rheological properties}

The colloidal dispersion is composed of cellulose nanofibrils (CNF) suspended in water. Cellulose nanofibrils were obtained by liberating fibrils from never dried sulfite softwood pulp (Domsjö Fabriker AB, Sweden) supplied by RISE (Research Institute of Sweden). Before defibrillation, never dried sulfite softwood pulp was subjected to TEMPO-mediated oxidation following the protocol described elsewhere [42,43]. Thereafter, defibrillation of the oxidized pulp was carried out by passing through a high pressure (1600 bars) Microfluidizer (M-110EH, microfluidics) with $400 / 200 \mu \mathrm{m}$ (one pass) and 200/100 $\mu \mathrm{m}$ (four passes) wide chambers connected in series. The resulting output is a cellulose nanofibril dispersion of $1 \mathrm{wt} \%$. Finally, a transparent colloidal dispersion of concentration $3 \mathrm{~g} \mathrm{dm}^{-3}$ was obtained by further dilution from $1 \mathrm{wt} \%$ to $0.3 \mathrm{wt} \%$ and 


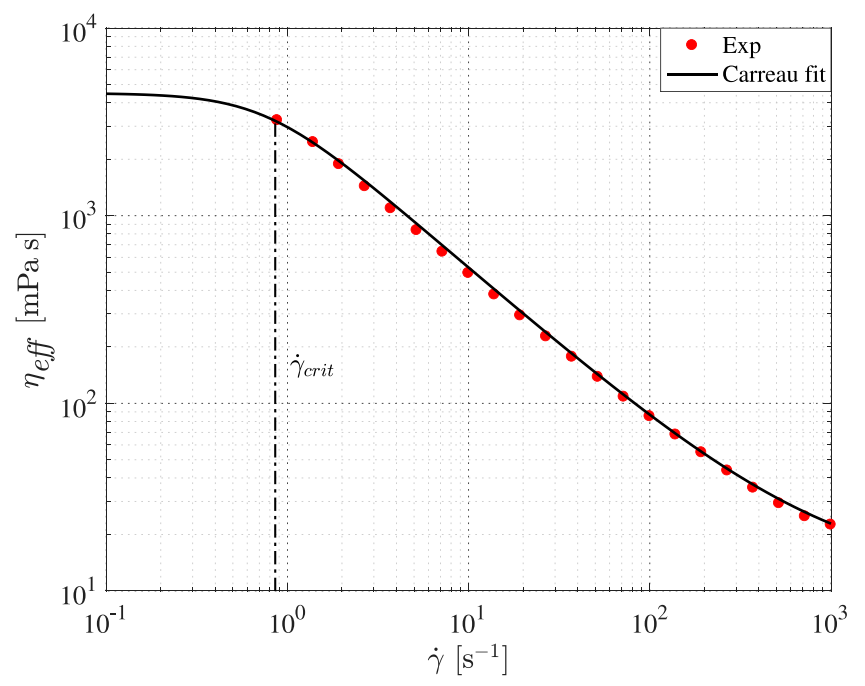

FIG. 2. Shear viscosity measurements of the colloidal dispersion represented by red dots. The solid black line represents the non-Newtonian Carreau model fit [see Eq. (3)], and the vertical dashed-dotted line denotes the critical shear rate $\dot{\gamma}_{\text {crit }}$ for the dispersion.

homogenization by an Ultra-Turrax dispersing tool (IKA, Sweden) for $10 \mathrm{~min}$ at 12000 revolutions per minute. The typical fibril lengths $L$ vary from $100-1500 \mathrm{~nm}$, and the average fibril diameter $d$ is $2.3 \pm 0.7 \mathrm{~nm}$ as determined by Atomic Force Microscopy (AFM, Dimension 3100 SPM, Veeco, USA) measurements.

The rheological characterization of the colloidal dispersion was performed using a bob and cup Kinexus pro+ rheometer (Malvern). This rheometer is well suited for accurate measurements of viscosity (indicated by red dots) over a range of shear rates, as observed in Fig. 2. The dispersion displays a non-Newtonian shear thinning behavior [44-46].

The rheological data can be described well by a Carreau model

$$
\eta_{\mathrm{eff}}=\eta_{\mathrm{inf}}+\left(\eta_{0}-\eta_{\mathrm{inf}}\right)\left[1+(\tau \dot{\gamma})^{2}\right]^{\frac{n-1}{2}}
$$

where $\eta_{\text {eff }}$ is the shear viscosity, $\eta_{\text {inf }}$ the infinite shear viscosity, $\eta_{0}$ the zero shear viscosity, $\tau$ the relaxation time, $\dot{\gamma}$ the shear rate, and $n$ the power index. The parameters of the Carreau model fit denoted by the solid black line in Fig. 2 are as follows: $\eta_{\text {inf }}=12 \mathrm{mPa} \mathrm{s}, \eta_{0}=4500 \mathrm{mPa} \mathrm{s}$, $\tau=1.306 \mathrm{~s}$, and $n=0.16$.

The shear thinning behavior is the consequence of microstructural rearrangements observed in the colloidal dispersion resulting from fibril and solvent molecular interactions, electrostatic interactions or due to effects of Brownian motion and fibre entanglement [47]. As the dimensions of the fibrils are very small, Brownian motion along with the entanglement effects is dominant over other interactions. At low shear rates, the microstructure of the fibrils are in disordered or isotropic arrangement. Once the shear rates are high enough to overcome Brownian effects, fibrils tend to reorganize by aligning and orienting toward the flow direction. As a result, the viscosity starts to decrease at a particular critical shear rate $\dot{\gamma}_{\text {crit }}$ indicating the onset of shear thinning. The critical shear rate can be obtained by taking the inverse of the orientational relaxation time of fibrils, $\dot{\gamma}_{\text {crit }}=\tau_{r}^{-1}$ where $\tau_{r}=1 /\left(6 D_{r}\right)$ [48]. The orientational relaxation time of a Brownian system can be estimated by calculating the rotational diffusion coefficient $D_{r}$, which is a measure of the rate at which a anisotropic system relaxes toward isotropy. 
The rotational diffusional coefficient for a fibril of length $L$ in a polydisperse system close to isotropy, could be approximated as [39,40,49,50]

$$
D_{r}(L) \approx \frac{\tilde{\beta} k_{B} T L_{*}^{4}}{\eta L^{7}},
$$

where $\tilde{\beta}$ is a numerical factor $\simeq 1000$ [51-53], the Boltzmann constant $k_{B}=1.38 \times 10^{-23} \mathrm{~J} \mathrm{~K}^{-1}$, the temperature $T=300 \mathrm{~K}$, the solvent viscosity $\eta_{s}=1 \mathrm{mPa}$. The entanglement length $L_{*}$ is defined as $[49,50]$

$$
L_{*}=\left(\int_{0}^{+\infty} \tilde{c}(L) L d L\right)^{-1 / 2},
$$

where $\tilde{c}$ is the concentration distribution dependent on fibril length $L$. The entanglement length $L_{*}$ indicates if the fibrils are in a dilute (not entangled, $c L^{3} \ll 1, c$ being concentration of fibrils, $\left.L<L_{*}\right)$ or semidilute regime $\left(1 \ll c L^{3} \ll L / d\right.$, entangled, $\left.L>L_{*}\right)$. More details related to $L_{*}$ can be found in Refs. [39,40]. For the present colloidal dispersion, $L_{*} \approx 45 \mathrm{~nm}$, and all the fibril lengths $L>L_{*}$, fall in the semidilute regime. Substituting all the values in Eq. (4), for an average fibril length $L \sim 750 \mathrm{~nm}$, the rotational diffusional coefficient close to isotropy becomes $D_{r} \approx 0.126 \mathrm{rad}^{2} \mathrm{~s}^{-1}$. Thus, $\dot{\gamma}_{\text {crit }}$ is approximated to be around $\dot{\gamma}_{\text {crit }}=6 D_{r} \simeq 0.80 \mathrm{~s}^{-1}$ as depicted in Fig. 2.

Thus, the low shear viscosity, i.e., zero shear viscosity $\eta_{0}$ of the colloidal dispersion is considered near to the critical shear rate $\dot{\gamma}_{\text {crit }}$ estimated as per the Eq. (4) (see Fig. 2). The viscosity ratio between the core and sheath flow defined as $\chi=\eta_{1} / \eta_{2}=4500$ is arrived based on the zero shear viscosity $\eta_{0}$ of the core fluid, i.e., $\eta_{1}=\eta_{0}=4500 \mathrm{mPa} \mathrm{s}$, and $\eta_{2}=1 \mathrm{mPa} \mathrm{s}$, being the viscosity of sheath fluid.

\section{Péclet number estimation}

To verify whether a de facto interface persists in the experimental system, timescale analysis is carried out by estimating the Péclet number based on the characteristic length scale $h$ of the channel system [5,27]. The translational diffusion coefficient $D$ for Brownian fibrils of average length $L \sim 750 \mathrm{~nm}$ and diameter $d \sim 2.3 \mathrm{~nm}$ diffused in a solvent of water is given as [48]

$$
D=\frac{k_{B} T \ln (L / d)}{2 \pi \eta l}
$$

where the temperature $T=300 \mathrm{~K}$, the Boltzmann constant $k_{B}=1.38 \times 10^{-23} \mathrm{~J} \mathrm{~K}^{-1}$, and the solvent viscosity $\eta=1 \mathrm{mPa}$ s. Substituting all the values, $D$ becomes approximately $5 \times$ $10^{-12} \mathrm{~m}^{2} \mathrm{~s}^{-1}$. The Péclet number is estimated as $\mathrm{Pe}=U h / D \approx 2 \times 10^{6}$ for an average flow velocity of $U \approx 10 \mathrm{~mm} \mathrm{~s}^{-1}$. Thus, as the Péclet number is very large, the timescale for the interdiffusion between the core fluid and sheath fluid is almost negligible compared to the convection timescale of the two fluids in the channel. Therefore, in such an event, a sharp de facto interface between the two fluids is likely to exist in the experiments $[3,5]$. Here, in the present experiments, a sharp de facto interface could exist only as long as the composition gradient of a fluid property is at hand, where EIT is expected to be present [8,41]. Moreover, the properties of such de facto interface can be associated to a distinct interface [2-5] between two immiscible fluids, wherein the two fluids, the core fluid (colloidal dispersion) and sheath fluid (water), could exhibit a near-immiscible behavior.

In addition, the rheological behavior of the colloidal dispersion is also controlled by the fact that the nanofibrils form a percolating volume spanning arrested state at very low concentrations [54]. This, in turn, makes the diffusion of nanofibrils into the surrounding sheath flows very slow compared to the timescales of the dynamics in the flow-focusing channel, making the Korteweg stresses long-lived. 


\section{Data acquisition method}

Three-dimensional core fluid thread topologies and the velocity field measurements are carried out by employing an light-based spectral domain optical coherence tomography. Optical coherence tomography (OCT) is a noninvasive volumetric imaging technique that uses a broadband light source, and operates based on the principle of low-coherence interferometry [55]. Utilising the Doppler principle, OCT can simultaneously capture the structural properties as well as the motion of opaque and turbid media sample with micron-level resolution [56,57]. The wavelength of the light source of the spectral domain OCT used in the present work is $1310 \mathrm{~nm}$ with a bandwidth of $270 \mathrm{~nm}$, and a resolution of $\sim 3 \mu \mathrm{m}$ in both the axial and transverse directions. More details related to the working principle of OCT, subsequent data acquisition and processing employed for the present study is described in Ref. [33].

In the present work, the 3D experimental measurements are performed for all the flow-focusing configurations illustrated in Fig. 1 with the above mentioned fluid properties. These experimental measurements will be used in Secs. III A and IIIC for cross-comparison with the numerical observations, and in Sec. III B to measure the experimentally acting $\Gamma_{e}$ between the colloidal dispersion-solvent system.

\section{B. Numerical setup}

The numerical computations have been performed by utilizing a recently developed finite volume based geometric volume of fluid (VoF) method, an interface advection algorithm called isoAdvector $[34,58]$. The implementation of the algorithm is incorporated in interIsoFoam, a two-phase incompressible immiscible open-source flow solver which is a part of the OpenFOAM community [35]. The algorithm accurately captures and advects the sharp interface, a key aspect in the numerical computation of multiphase flows. The rationale behind choosing the immiscible fluid solver was elucidated in the introduction, and will be again clarified in the upcoming Sec. V. The set of equations being solved for an immiscible system of two fluids are the continuity equation

$$
\nabla \cdot \boldsymbol{U}=0
$$

and the Navier-Stokes equation together with the continuum representation of an interfacial tension force $\boldsymbol{F}_{\boldsymbol{s}}[59]$,

$$
\begin{gathered}
\frac{\partial \rho_{b} \boldsymbol{U}}{\partial t}+\nabla \cdot\left(\rho_{b} \boldsymbol{U} \boldsymbol{U}\right)=-\nabla p+\nabla \cdot \boldsymbol{T}+\boldsymbol{F}_{\boldsymbol{s}}, \\
\boldsymbol{F}_{\boldsymbol{s}}=\gamma \kappa(\nabla \alpha),
\end{gathered}
$$

where $\gamma$ is the interfacial tension and $\kappa$ is the curvature of the interface

$$
\kappa=-\nabla \cdot\left(\frac{\nabla \alpha}{|\nabla \alpha|}\right)
$$

and the equation for the advection of phase and volume fraction $\alpha$

$$
\frac{\partial \alpha}{\partial t}+\nabla \cdot(\alpha \boldsymbol{U})=0 .
$$

Here $\boldsymbol{T}$ represents the deviatoric stress tensor, $\boldsymbol{U}$ is the velocity vector field and $p$ is the pressure field. The density $\rho_{b}$ and viscosity $\mu_{b}$ are computed as $\rho_{b}=\rho_{1} \alpha+\rho_{2}(1-\alpha), \mu_{b}=\mu_{1} \alpha+\mu_{2}(1-$ $\alpha$ ) based on the weighted average distribution of the volume fraction $\alpha$ of fluid where $\rho_{1}, \rho_{2}, \mu_{1}, \mu_{2}$ are the densities and the viscosities of the two fluids, respectively.

In the present study, 3D numerical computations are performed for the geometrical configurations illustrated in Fig. 1. At the channel walls, a no-slip velocity boundary condition and a contact angle of $\theta=0^{\circ}$ is imposed for the phase field. A uniform velocity flow profile is prescribed at the core and sheath flow channel inlets based on the flowrates of $Q_{1}=6.5 \mathrm{~mm}^{3} \mathrm{~s}^{-1}$ and 
$Q_{2}=7.5 \mathrm{~mm}^{3} \mathrm{~s}^{-1}$. At the channel outlet, pressure is set to atmospheric, and zero gradient for the volume fraction. The non-Newtonian Carreau model depicted in Fig. 2 is implemented for the rheology of the core fluid while the viscosity of water $\eta_{2}=1 \mathrm{mPa}$ s is set for the sheath fluid.

The numerical computations are performed on Cartesian meshes. The size of the computational domain comprises of three inlet channels of length $5 h$ and an outlet channel of length $30 h$ with a square cross-section of width $h=1 \mathrm{~mm}$. The inlet channel domains are discretized with a unidirectional grid along the channel length and an equidistant grid spacing across the cross-section. The outlet channel domain has an equidistant grid spacing of $\Delta=2 \times 10^{-5} \mathrm{~m}(\Delta x=\Delta y=\Delta z)$ both along the channel length and across the square cross-section. An adaptive time step method is used to achieve the stability and convergence of the computations. The computations were run on 256 processors, and the simulations took 48 hours. For additional details concerning to numerics and grid convergence, we guide the interested reader to look upon Ref. [33].

In application to two-phase microfluidic flows via hydrodynamic focusing, and to ensure the immiscible solver captures the actual capillary effects, the solver has been thoroughly tested and validated through an extensive comparison, by reproducing a state space map based on the capillary numbers of two fluids with previous experimental studies [60]. As detailed in Ref. [33], the simulations performed with a pair of Newtonian immiscible fluids demonstrate very good agreement with the experiments [60], capturing diverse flow patterns such as threading, jetting, dripping and tubing regimes typically observed in a microfluidic flow-focusing channels. In addition, excellent qualitative and quantitative agreement between numerical and experimental results [33] is demonstrated.

\section{RESULTS AND DISCUSSION}

We first carry out a detailed numerical and experimental investigation of the effect of confluence angle $\beta$ on the 3D thread morphology and shape of the wetted region. Then, in Sec. III B, experimentally measured thread height $\varepsilon_{z} / h$ as in the case of reference flow-focusing configuration $\left(\beta=90^{\circ}\right)$ is compared with the master curve to estimate the $\Gamma_{e}$ acting between the present experimental fluids.

The flow rates and rheologies of the core and sheath fluids in the computations and experiments are set as mentioned earlier in Secs. IIA1 and IIA2.

\section{A. Morphology of the thread and shape of the wetting region}

In this section, we compare the 3D experimental thread shape measured with OCT for geometrically varying flow-focusing configurations described in Sec. II A, and those obtained by numerical computations for the corresponding configurations. All the numerical computations are performed with an ultralow interfacial tension $\gamma=0.615 \mathrm{mN} \mathrm{m}^{-1}$ modeling EIT. This choice will be motivated in the upcoming Sec. III B.

First, a few generic features applicable to all the geometrical configurations are noted before moving into a geometry specific detailed discussion. Figures 3(a)-3(e) show the experimental and numerical 3D thread shapes of the colloidal dispersion for various confluence angles $\beta$. The qualitative agreement between experiments and numerics is ascertained to be quite good. A closer view shows the core and sheath flow channel walls intersect at the point of confluence corresponding to $x / h=0$. The region beginning from $x / h=0$ to the position where the confluence of core and sheath flow channels end (indicated by dashed magenta lines) is referred to as the confluence region. However, the length of confluence region varies depending on the confluence angle $\beta$. The confluence region is shortest $(0 \leqslant x / h \leqslant 1)$ for the reference configuration $\left(\beta=90^{\circ}\right)$ and longest $(0 \leqslant x / h \leqslant 2)$ for $\beta=\left[30^{\circ}, 150^{\circ}\right]$ pair. Meanwhile, at the top and bottom walls of the channel (i.e., at $z / h= \pm 0.5$ ), the colloidal dispersion invariably remains attached to the walls even after $x / h>0$. The dispersion continues to stay attached to the upper and lower walls up to a pinch-off point. The region originating from $x / h=0$ to the colloidal thread detachment point is denoted the wetted region and the subsequent length is called wetted length $L_{w} / h$ [see Fig. 4(a)]. Indeed, 


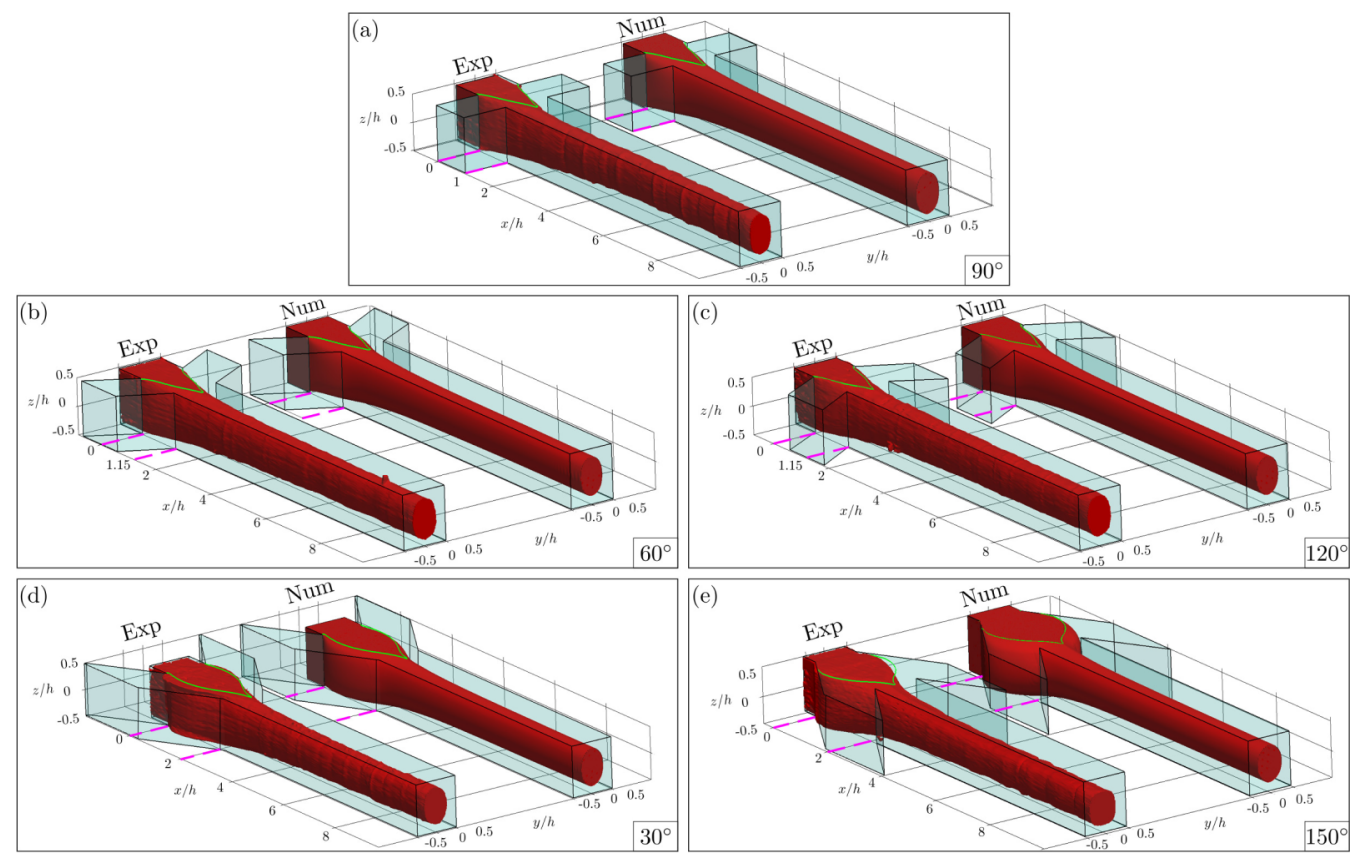

FIG. 3. (a)-(e) 3D view of the experimental and numerical threads for flow-focusing configurations with varying confluence angle $\beta$. The green curves at the top planes $z / h=0.5$ on all the five panels represent the boundary of wetted region created by the core fluid before the detachment. The horizontal dashed magenta lines in all the five panels indicate the confluence region for the respective configurations.

both the wetted region and the wetted length vary with $\beta$ as observed in Figs. 3(a)-3(e) (marked by green curves at the top planes $z / h=0.5$ ), and more clearly in Figs. 4(a)-4(e). Also here, a very good agreement is notable between the computations and experiments.

Further, as observed from Figs. 3(a)-3(e), the evolution of streamwise thread development appears to be affected by $\beta$ in the upstream while at far downstream the thread shapes seem to be nearly elliptical. The cross-sectional views of the elliptical thread at downstream position $x / h=10$ are displayed in Figs. 5(a)-5(e) with its major and minor axes denoted by $\varepsilon_{z} / h, \varepsilon_{y} / h$. Besides, quantitatively, the excellent agreement is exemplified between experimentally measured height $\varepsilon_{z} / h$ (red curves) and width $\varepsilon_{y} / h$ of the thread (blue curves) with the numerically computed thread height and width (denoted by various color curves as per the respective configuration) depicted in Figs. 6(a)-6(e).

In view of the above observations, it is apparent that the confluence angle $\beta$ has a significant influence both on the wetted region morphology and the thread development. Indeed, characterising the influence of $\beta$ is an important aspect from the hydrodynamic assembly of nanofibrils point of view [37], since the shape of thread regulates the cross-section of an assembled material. This, in turn, could enhance the scope for synthesizing materials of complex shapes such as rods, ellipsoids, discs and so on. Hence, in what follows, is a systematic analysis of the effect of confluence angle $\beta$.

Beginning with the reference configuration $\left(\beta=90^{\circ}\right)$, the sheath flows are perpendicular to the core flow. Accordingly, sheath flow momentum is acting only normal to the core flow over the confluence region $0 \leqslant x / h \leqslant 1$, and the sheath fluid impinges the core fluid with maximum impact in the normal direction as seen in Fig. 3(a). As a result, the colloidal thread detaches with a shorter wetted length $L_{w} / h \approx 1.8$ [Fig. 4(a)] compared to other configurations [Figs. 4(b)-4(e)]. In fact, as it can be seen from Fig. 6(a), even the thread width $\varepsilon_{y} / h$ decreases much faster than the height $\varepsilon_{z} / h$ up to $x / h \approx 6$, highlighting the extent of impact of sheath flow momentum along the streamwise 

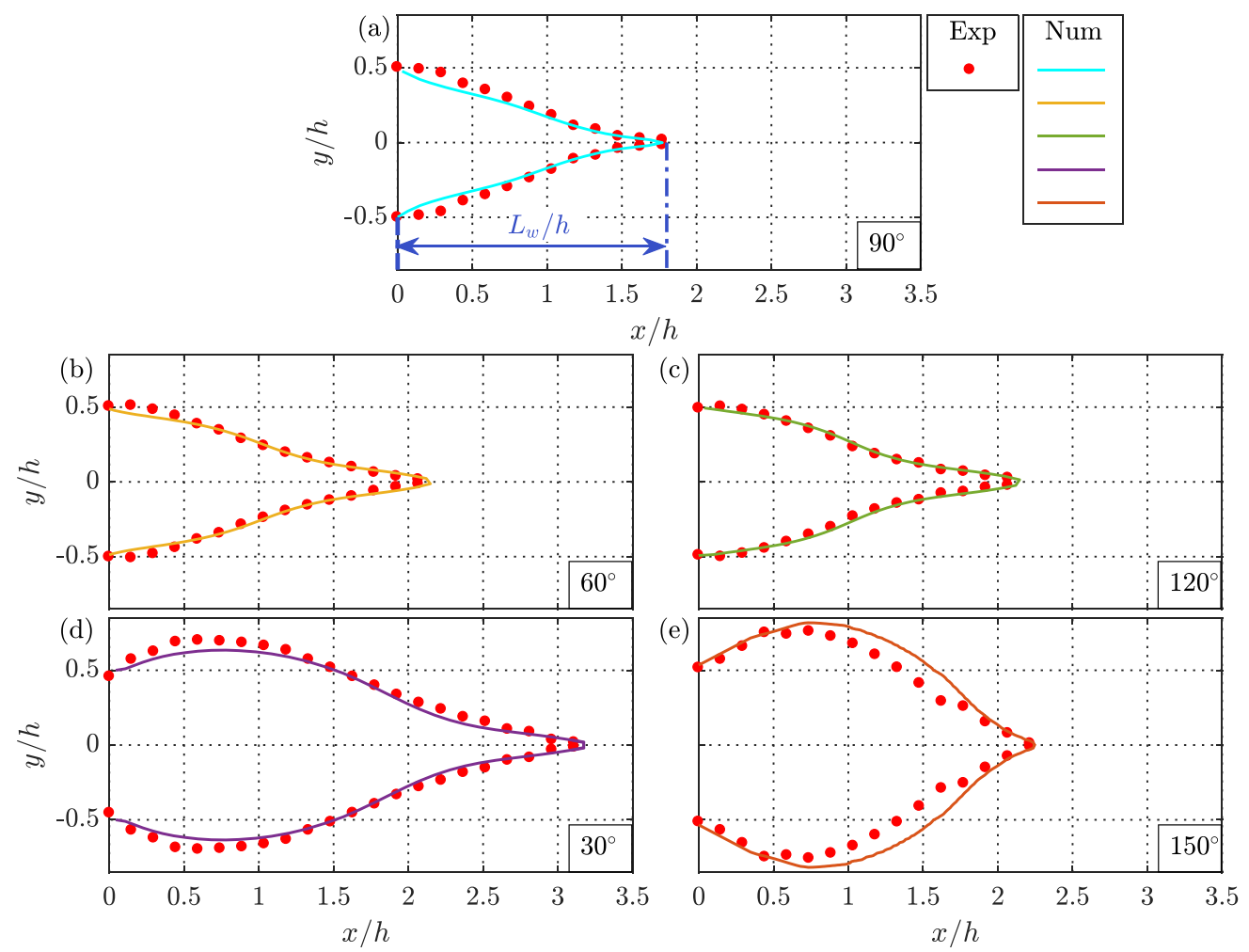

FIG. 4. (a)-(e) Top view of the experimental and numerical wetted region morphologies of the core fluid in the plane $z / h=0.5$ for flow-focusing configurations with varying confluence angle $\beta$. The distance from $x / h=$ 0 to the point of detachment of core fluid from the top wall is referred to as 'wetted length' $L_{w} / h$, indicated in panel (a). The wetted region shape and length $L_{w} / h$ vary as per the respective geometrical configuration. These wetted boundaries are also shown in the previous Figs. 3(a)-3(e) at the top plane $z / h=0.5$ marked with green curves.

thread development. Far downstream the width attains a constant value. However, after the thread detachment, the effect of sheath flow momentum on the height is less significant. Furthermore, since the sheath flow momentum is maximized in the direction normal to the core flow when $\beta=90^{\circ}$, all the upcoming geometrical configuration discussions will be relative to this reference configuration. In particular when the sheath flow momentum normal to the core flow is taken into consideration, and hence the choice of geometrical configurations placement in the order as shown in Fig. 1 were opted.

Continuing to the $\beta=\left[60^{\circ}, 120^{\circ}\right]$ pair of configurations, the sheath flow momentum acts both in parallel and normal to the streamwise core flow at their respective confluence regions as seen in Figs. 3(b) and 3(c). Most importantly, both the configurations have the same length of confluence regions, i.e., $0 \leqslant x / h \leqslant 1.15$. However, in the $\beta=60^{\circ}$ case, the sheath flow is impinging the core flow in the same direction as the streamwise core flow, which can be referred to as positive impingement, while in the $\beta=120^{\circ}$ case, the sheath flow is impinging opposite to the direction of the streamwise core flow, i.e., a negative impingement. For the purpose of clarity, top views of both the configurations illustrated in Figs. 1(b) and 1(c), gives a better notion of positive and negative impingement (following the signs of arrow representing the core and sheath flows). Surprisingly, the thread morphology and shape of the wetted region for these two cases closely resembles each other as seen in Figs. 3(b), 4(b) and 3(c), 4(c) in spite of difference in the fundamental impingement 

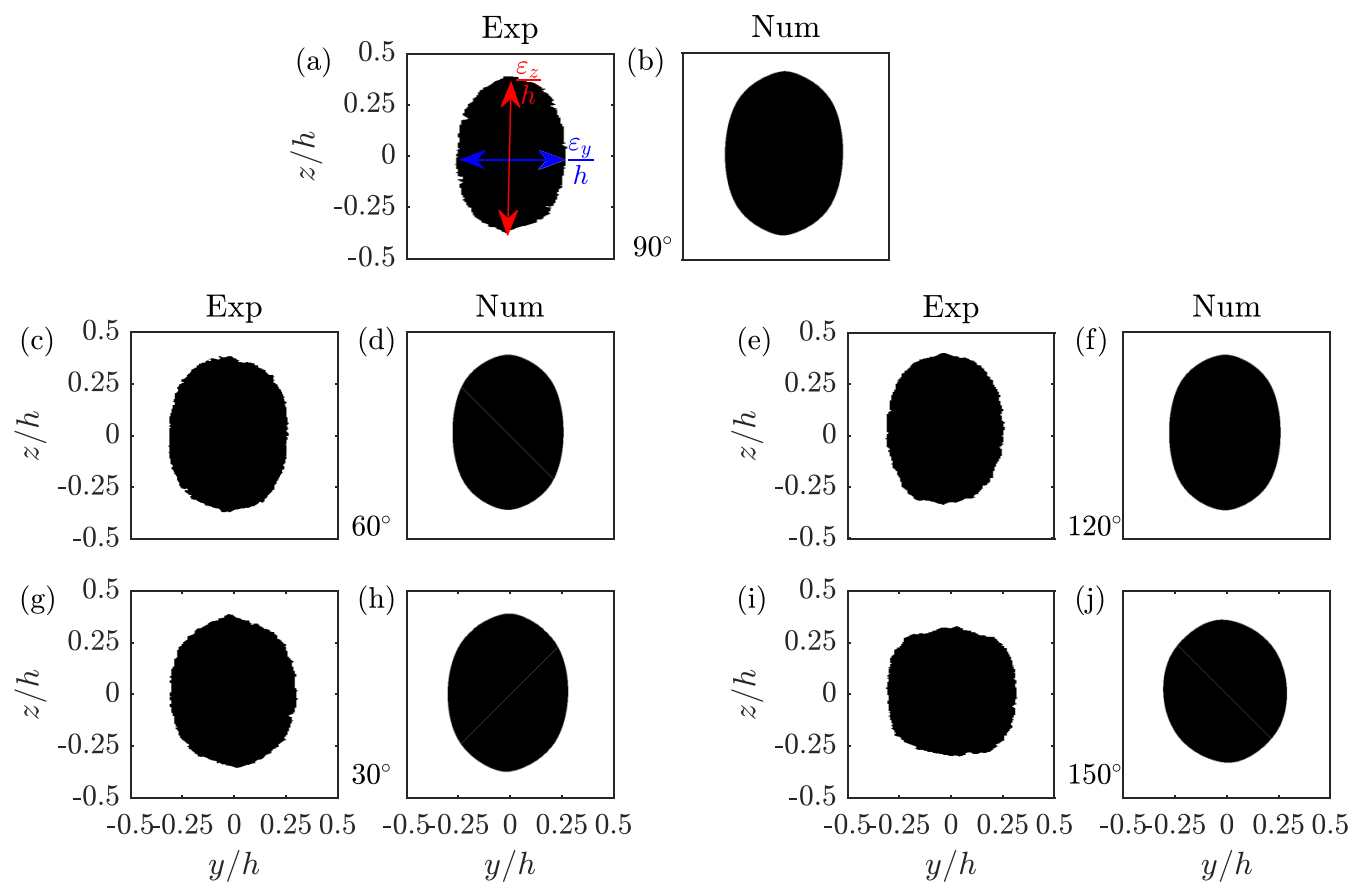

FIG. 5. Experimental [panels (a), (c), (e), (g), and (i)] and numerical [panels (b), (d), (f), (h), and (j)] thread cross-sections at downstream position $x / h=10$ for flow-focusing configurations with varying confluence angle $\beta$. Black color denotes the core fluid while white color represents the sheath fluid. Thread width $\varepsilon_{y} / h$ (blue) and height $\varepsilon_{z} / h$ (red) denoting the ellipsoid axes is pictured in panel (a).

direction. As it can be observed from Figs. 4(b) and 4(c), in both the cases, the wetted area increases and the wetted length extends up to $L_{w} / h \approx 2.1$, which is much longer than the reference configuration $L_{w} / h$ [Fig. 4(a), $L_{w} / h \approx 1.8$ ]. This indicates that the main effect of $\beta$ is through the sheath flow momentum normal to the core flow, which has been weakened by about $13.4 \%$ in relative to the reference configuration in both cases. Further, during the development of the thread width $\varepsilon_{y} / h$ and height $\varepsilon_{z} / h$ as a function of downstream positions $x / h$, both the configurations exhibit similar characteristics as displayed in Figs. 6(b) and 6(c). In both cases, the width and height of the thread decay slightly faster up to $x / h \approx 6$ and thereafter, stays almost constant far downstream with an elliptical cross-section as visualized in Figs. 5(c), 5(d) and 5(e), 5(f).

However, for the $\beta=\left[30^{\circ}, 150^{\circ}\right]$ pair, the thread shape and wetted region morphologies differ substantially as viewed in Figs. 3(d), 4(d) and 3(e), 4(e). Figures 3(d) and 3(e) show the length of confluence regions $0 \leqslant x / h \leqslant 2$ being larger than in other configurations [Figs. 3(a) or 3(b), 3(c)]. Notably, in both cases, there is an expansion of the colloidal thread symmetrically in the transverse or sheath flow direction ( $y$ direction) near the confluence region. This could be due to the decrease in the impact of sheath flow momentum normal to the core flow. Compared to the reference configuration, the magnitude of normal sheath flow momentum is reduced by around $50 \%$ due to the effect of $\beta$. In the case of positive impingement of sheath flow $\left(\beta=30^{\circ}\right)$ there is a small expansion of the wetted area first and the wetted length extends up to $L_{w} / h \approx 3.1$ as depicted in Fig. 4(d). However, for the negative impingement $\left(\beta=150^{\circ}\right)$, the wetted area expands considerably by curving outward in the transverse direction with a much shorter wetted length $L_{w} / h \approx 2.1$ as seen from Fig. 4(e).

The evolution of streamwise thread development in Figs. 6(d) and 6(e) shows that the width $\varepsilon_{y} / h$ of the thread in the confluence region $0 \leqslant x / h \leqslant 2$ overshoots up to $\varepsilon_{y} / h \approx 1.5$ ( $\beta=30^{\circ}$ case $)$ and 


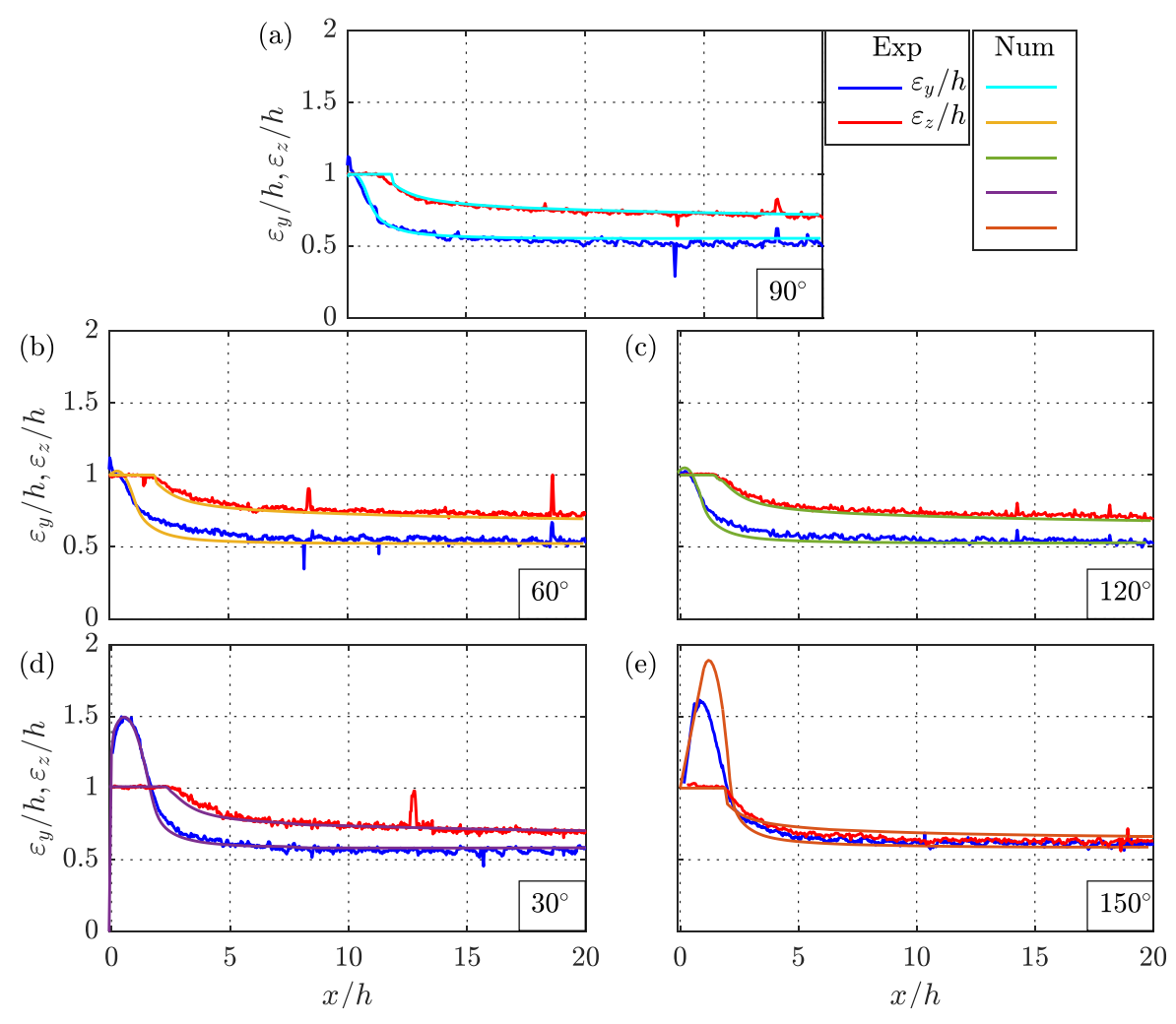

FIG. 6. (a)-(e) Thread width $\varepsilon_{y} / h$ and height $\varepsilon_{z} / h$ as a function of downstream positions $x / h$ for flowfocusing configurations with varying confluence angle $\beta$. The experimental data are represented in blue and red color, while the numerical data are denoted by various solid color lines shown in the rightmost legend in panel (a) as per the respective geometrical configuration.

even more for the negative impingement $\left(\beta=150^{\circ}\right)$ before the decay, highlighting the expansion of the thread in the tranverse direction. Another striking behavior is for the $\beta=150^{\circ}$ configuration, where the experimental cross-section of the thread is somewhat different from the elliptical shape as illustrated in Figs. 5(i) and 5(j). In fact, the numerical curve shows an overprediction in the upstream near the confluence junction during the expansion. This feature of experimental thread cross-section in Fig. 5(i) is not accurately captured by the numerics.

\section{B. Estimation of effective interfacial tension}

In this section, we first present an overview of the model used to obtain a master curve that was proposed at the conclusion of our previous study in Ref. [33]. Then, we fit the master curve to an exponential function, and employ it here to estimate the $\Gamma_{e}$ between the present experimental fluids.

Figure 7(a) summarizes our observations performed through an extensive set of numerical computations in Ref. [33]. These computations were performed with the reference flow-focusing setup for a set flow rate and the given rheology of the fluids. As mentioned in the introduction, employing an immiscible fluid solver, we had demonstrated that soon after the core fluid detachment from the top and bottom channel walls of the channel at $x=L_{w}$, the thread height varied from noncircular (near-ellipsoidal) cross-section to a circular shape over a range of interfacial tensions $0.024 \mathrm{mN} \mathrm{m}^{-1}<\gamma<3.000 \mathrm{mN} \mathrm{m}^{-1}$. The larger the value of $\gamma$, the faster the thread converges toward a circular shape for which the thread height attains a constant value of $\varepsilon_{z} / h \sim 0.63$. 

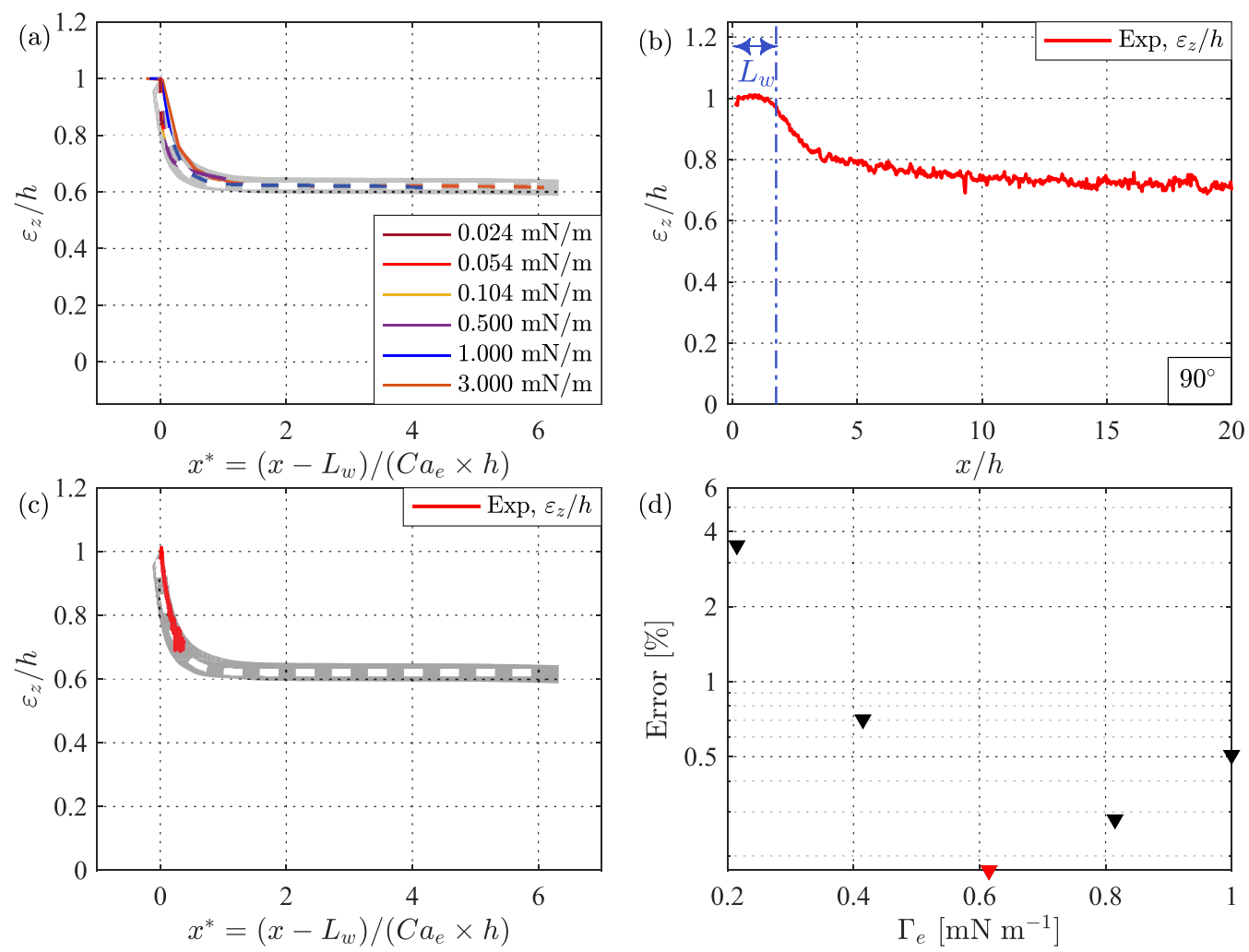

FIG. 7. (a) The numerical thread heights $\varepsilon_{z} / h$ at various interfacial tensions $\gamma$ plotted as a function of scaled downstream length $x^{*}$ (detailed in text). The data collapse on to a master curve. These simulated data are taken from Ref. [33]. The master curve can be best represented by an exponential fit [see Eq. (14)] denoted by the dashed-white line. The fit parameters $a, b$, and $c$ are reported in Table I. Panel (b) shows the experimental thread height $\varepsilon_{z} / h$ as a function of downstream positions $x / h$ measured in the reference configuration $\left(\beta=90^{\circ}\right)$ of the present study. The dash-dotted vertical blue line in panel (b) indicates the measured wetted length of the thread before the detachment $\left(L_{w} / h \sim 1.8\right)$. In panel (c), the experimental thread height $\varepsilon_{z} / h$ is compared with the exponential fit (dashed-white line) representing the master curve to estimate the $\Gamma_{e}$ acting between the present experimental fluids. The light gray backdrop in panels (a) and (c) is drawn for the purpose of better visualization of the plotted data. Panel (d) shows the percentage variation of the error (defined in text) between the numerical and experimental thread heights for a range of $\Gamma_{e}$ values near to the estimated $\Gamma_{e}$ (Table I) obtained from the master curve fit in panel (c). The red marker in panel (d) indicate the $\Gamma_{e}$ that gives the best match with the experimental measurement.

Following Ref. [33], using appropriate notations, the typical time and length scales for a nearellipsoidal thread to approach circular shape can be written as

$$
\begin{gathered}
\tau=\frac{\eta_{1}}{\delta P} \propto \frac{\eta_{1}}{\gamma}, \\
l_{r}=U \tau \propto \frac{\eta_{1} Q_{1}}{\gamma h^{2}},
\end{gathered}
$$

where $U, Q_{1}$, and $\eta_{1}$ are the velocity, flow rate, and dynamic viscosity of the core fluid. $\delta P$ is the pressure gradient dependent on the thread geometry and is proportional to the interfacial tension $\gamma$. Comparing Eqs. (2) and (13), $\eta_{1} Q_{1} /\left(\gamma h^{2}\right) \simeq \eta Q /\left(\Gamma_{e} h^{2}\right)=\mathrm{Ca}_{e}$, the effective capillary number 
TABLE I. Fitting parameters $a, b$, and $c$ of the exponential fit [Eq. (14), dashed-white line in Figs. 7(a) and $7(\mathrm{c})$ ] representing the master curve. The estimated $\Gamma_{e}$ acting between the present experimental fluids is obtained by utilizing the spatially measured experimental thread height $\varepsilon_{z} / h\left(\beta=90^{\circ}\right)$ [shown in Fig. 7(b)] and Eq. (14) along with the fit parameters, and other experimentally acquired quantities such as viscosity $\left(\eta_{1}\right)$, flow rate $\left(Q_{1}\right)$ of the core fluid and the channel width $h$.

\begin{tabular}{lccc}
\hline \hline$a$ & $b$ & $c$ & Estimated $\Gamma_{e}\left(\mathrm{mN} \mathrm{m}^{-1}\right)$ \\
\hline $0.240 \pm 0.0008$ & $2.950 \pm 0.015$ & $0.622 \pm 0.0006$ & $0.756 \pm 0.0005$ \\
\hline \hline
\end{tabular}

where the numerical interfacial tension $\gamma$ models the "effective" interfacial tension in experiments, i.e., $\gamma \equiv \Gamma_{e}$.

Thus, from Eqs. (12) and (13), a scaled downstream length $x^{*}$ can be obtained by renormalizing the downstream length $\left(x-L_{w}\right)$ with $l_{r}$ leading to $x^{*}=\left(x-L_{w}\right) /\left(\mathrm{Ca}_{e} \times h\right)$, respectively. As depicted in Fig. 7(a), all the simulated thread heights $\left(\varepsilon_{z} / h\right)$ at various interfacial tensions $\gamma$ collapse well on a master curve when plotted with the scaled coordinate $x^{*}$.

The master curve depicted in Fig. 7(a) can be best fitted to an exponential function denoted by dashed-white line with the fitting parameters $a, b$, and $c$ given in Table I:

$$
\varepsilon_{z} / h=a \times e^{-b x^{*}}+c .
$$

The light gray backdrop in Figs. 7(a) and 7(c) is in place solely for the sake of clarity in the graphics of the plots. The fit parameters are evaluated through nonlinear least-square regression performed in Matlab.

To estimate the $\Gamma_{e}$ acting between the present colloidal dispersion - solvent system, we use the spatial evolution of thread height $\varepsilon_{z} / h$ measured experimentally with the reference flow-focusing configuration $\left(\beta=90^{\circ}\right)$ as depicted in Fig. 7(b), and compare with the exponential fit representing the master curve as shown in Fig. 7(c). By solving Eq. (14) together with the fit parameters reported in Table I, the scaled downstream length $x^{*}$ is obtained. As noted from above, the expression for $x^{*}$ is given as $x^{*}=\left(x-L_{w}\right) /\left(\mathrm{Ca}_{e} \times h\right)$. Substituting all the experimentally measured variables in $x^{*}$ and $\mathrm{Ca}_{e}$ such as the core fluid flow rate $Q_{1}=6.5 \mathrm{~mm}^{3} \mathrm{~s}^{-1}$, viscosity of the core fluid, $\eta_{1}=4500 \mathrm{mPa} \mathrm{s}$, the wetted length $L_{w} \sim 1.8 \mathrm{~h}$ along with the streamwise downstream positions $x$ of the thread height, and the channel width $h$, the estimated $\Gamma_{e} \simeq 0.765 \mathrm{mN} \mathrm{m}^{-1}$ can be retrieved.

Furthermore, as a verification, the numerical computations were performed in close proximity to the estimated $\Gamma_{e}$ utilizing the reference flow-focusing configuration $\left(\beta=90^{\circ}\right)$. The difference between the numerical and experimental [Fig. 7(b)] thread height is plotted in Fig. 7(d) as an error, which is defined as

$$
\delta_{\varepsilon_{z}}\left(\Gamma_{e}\right)=\sum_{i=1}^{N}\left|\frac{\varepsilon_{z, i}^{n u m}-\varepsilon_{z, i}^{\exp }}{\varepsilon_{z, i}^{\exp }}\right|^{2},
$$

where $i=1$ to $N$ are the downstream positions at which thread heights $\varepsilon_{z} / h$ are evaluated after the detachment of thread from the top and bottom channel walls. The minimum of $\delta_{\varepsilon_{z}}\left(\Gamma_{e}\right)$ indicated by a filled red symbol in Fig. 7(d) occurs at $\Gamma_{e}=0.615 \mathrm{mN} \mathrm{m}^{-1}$ which, in turn, affirms the estimated $\Gamma_{e}$ to be good with fairly accurate order of magnitude. This value of $\Gamma_{e}=\gamma$ is used in the numerical simulations of all the geometrically varying flow-focusing setups as discussed in the previous Sec. III A.

An interesting observation worth noting here is, that the value of $\Gamma_{e}\left(\mathcal{O} \sim 10^{-1} \mathrm{mN} \mathrm{m}^{-1}\right)$ for the present study colloidal dispersion-solvent system is almost one decade higher in magnitude than our previously evaluated value $\left(\mathcal{O} \sim 10^{-2} \mathrm{mN} \mathrm{m}^{-1}\right)$ for a similar, but not identical colloidal dispersion-solvent system [33] at the same dispersion concentration $(0.3 \mathrm{wt}$. \%). Both the colloidal dispersions exhibit non-Newtonian shear thinning behavior, and the zero shear viscosity for the present case is $\eta_{1}=\eta_{0} \simeq 4500 \mathrm{mPa}$ s while it was $1750 \mathrm{mPa}$ s in Ref. [33]. The order of magnitude 
difference in $\Gamma_{e}$ at same dispersion concentration could be attributed to the variation in length fraction of nanofibrils, interfibril interactions leading to different rheological properties of the colloidal dispersion, in turn to that on variant dispersion-solvent de facto interface properties through different $K$ in Eq. (1). At this point, it is also worth commenting that the EIT between a very dilute dispersion and its own solvent will be very weak due to the fact that $\Gamma_{e} \sim \Delta \Phi^{2}$.

Indeed, the above observations concur with the experimental studies performed by Refs. [8,41], where $\Gamma_{e}$ between colloidal dispersions and its own solvent were measured, and the variations in $\Gamma_{e}$ span over 5 decades $\left(10^{-4}-10^{1} \mathrm{mN} \mathrm{m}^{-1}\right)$ for mild changes in the concentration.

Given the non-Newtonian nature of the colloidal dispersion, and the dispersion being subjected to extensional flow, one could expect that extensional viscosity could play a role in the dynamics of the system. However, for this particular set of experimental fluids, i.e., a complex fluid made of cellulose nanofibrils suspended in water, shear-dependent viscosity (see Fig. 2, Sec. IIA2) together with an ultralow interfacial tension modeling the EIT between the dispersion and its own solvent seem to be sufficient to describe the spatial evolution of threads quite well. This is evident from the master curve [Figs. 7(a) and 7(c)] obtained through a simple scaling model [Eqs. (12) and (13)] where the "effective" capillary number $\left(\mathrm{Ca}_{e} \simeq \eta_{1} Q_{1} /\left(\Gamma_{e} h^{2}\right)\right)$ controls the thread evolution. In other words, the low or zero shear viscosity $\left(\eta_{1}=\eta_{0}\right)$ of the core fluid, and interfacial tension modeling EIT describes very well the spatial development of the thread for a given core fluid flow rate $Q_{1}$ and channel width $h$. In addition, as seen from the previous Sec. III A, for a set flow rate, utilizing the shear-dependent rheology (see Fig. 2) described by a non-Newtonian Carreau model and a constant value of effective interfacial tension $\left(\gamma=\Gamma_{e}=0.615 \mathrm{mN} \mathrm{m}^{-1}\right)$, the experimentally observed 3D thread shapes and their spatial developments are very well captured and reproduced numerically for a diverse set of geometrically varying flow-focusing configurations. Therefore, there is no indication of significant effects from other hydrodynamic phenomena on the thread evolution for this system.

\section{Centreline velocities}

In addition to the evolution of thread shape, quantitative understanding of the flow field behavior is vital for the modeling and prediction of hydrodynamic alignment of nanofibrils [61]. Alignment of nanofibrils is a key factor in controlling and tuning the material properties [62] of assembled materials.

Figure 8 shows the velocity variation along the centreline of the colloidal thread as a function of downstream positions $x / h$ for all the five confluence angle geometries. The agreement between numerical computations and experimental measurements is excellent. Dashed vertical lines (magenta) in all the panels [Figs. 8(a)-8(e)] mark the confluence regions. The trend of the centerline velocity $\left(U_{c}\right)$ variation is more or less similar among all configurations. First, the velocity is constant in the inlet channel before the confluence region $(x / h<0)$. There is a slight deceleration right after the confluence point at $x / h=0$ that is followed by a rapid acceleration before a high steady value at far downstream positions $(x / h \geqslant 10)$ is reached. However, a careful inspection of Figs. 8 and 9 unveils a considerable variation in the minimum and maximum centreline velocity, degree of deceleration and acceleration for different confluence angles $\beta$. These variations will now be studied in detail.

In the case of the reference configuration $\left(\beta=90^{\circ}\right)$, close to the confluence point $x / h \simeq 0$, the deceleration is ascertained to be small as observed from Figs. 8(a) (confluence region) and 9(c) (cyan color). As a consequence, the minimum centreline velocity $U_{c, \text { min }}$ is higher (meaning lower deceleration) than the other configurations as seen from Fig. 9(a). Subsequent to deceleration, a substantial increase in the velocity caused by the sheath flow momentum normal to the core flow leads to acceleration of the core flow. As depicted in Fig. 9(c), $\dot{\varepsilon}$ (cyan color) continues to increase till the end of the confluence region $(x / h \sim 1)$, and thereafter, $\dot{\varepsilon}$ decays to 0 at around $x / h \approx 5$ since the velocity is almost constant as observed in Fig. 8(a). However, far downstream, as seen in Fig. 9(b), $U_{c, \max }$ is lowest as compared to all the other configurations.

For the $\beta=\left[60^{\circ}, 120^{\circ}\right]$ pair, the velocity behavior duplicate each other as observed from Figs. 8(b) and 8(c) and more clearly from the strain rate $\dot{\varepsilon}$ plot in Fig. 9(c). It is worth to recall 

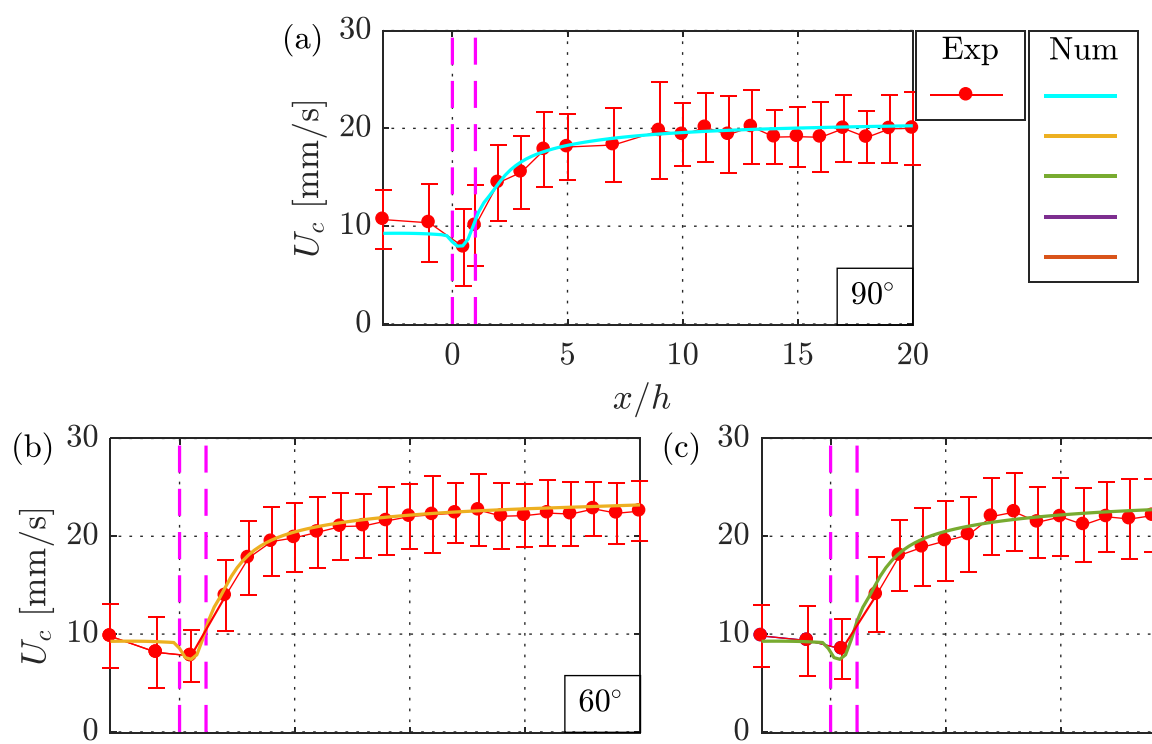

(c)
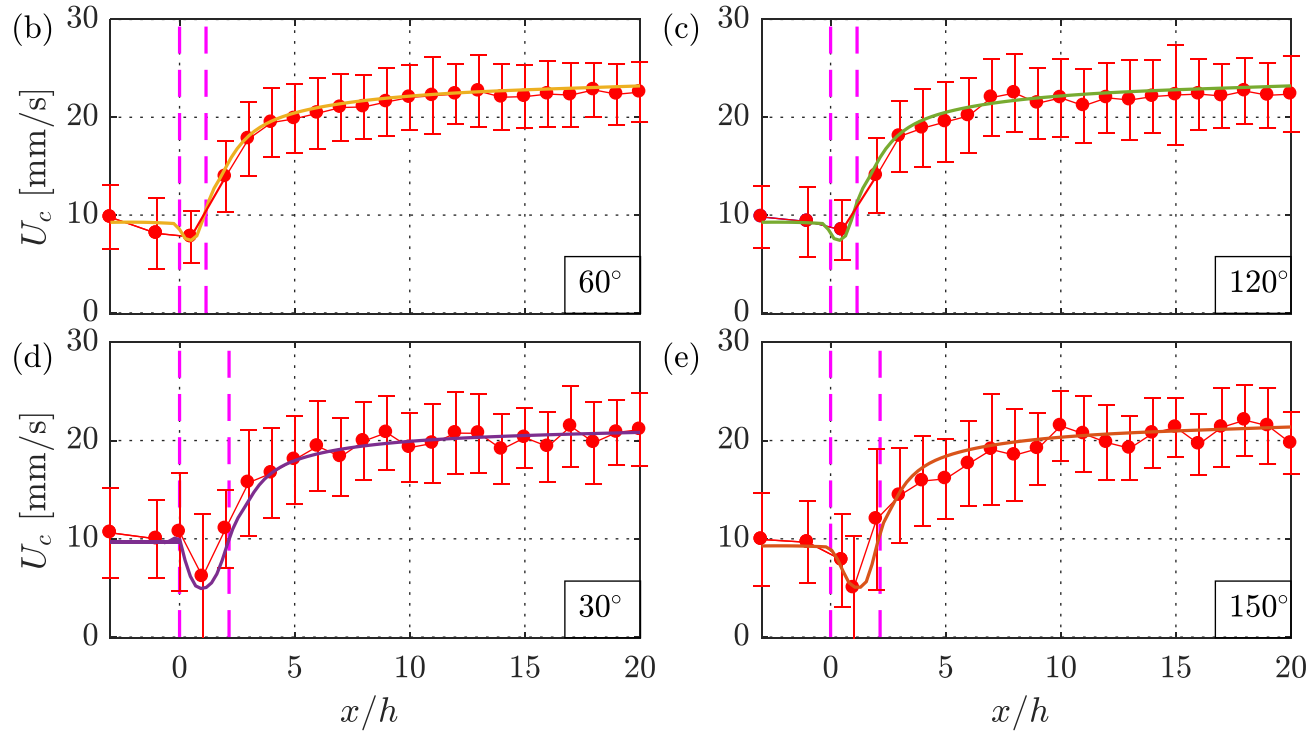

(e)

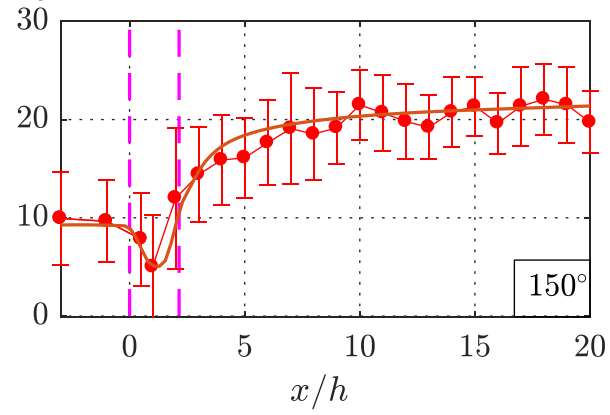

FIG. 8. Panels (a)-(e) show the experimental and numerical centreline velocity $U_{c}$ as a function of streamwise downstream positions $x / h$ for flow-focusing configurations with varying confluence angle $\beta$. The vertical dashed lines (magenta) on all the panels denote the confluence region of the respective configurations. Error bars represent the standard deviation of experimental measurements at each position.

that this similarity of the flow mirrors the similarity of the thread shape evolution as seen earlier from Figs. 3(b), 3(c) to Figs. 6(b), 6(c). In both cases, the effect of $\beta$ appends the streamwise flow through the contribution by sheath flow momemtum acting parallel to the core flow. As a result, the core fluid velocity increases much faster than the reference configuration velocity. For both cases, $U_{c, \min }$ and $U_{c, \max }$ are symmetric with respect to $\beta=90^{\circ}$ as seen in Figs. 9(a) and 9(b). However, $U_{c, \min }$ is lower for the $\beta=90^{\circ}$ case (implying higher deceleration) and $U_{c, \text { max }}$ is highest for $\left[60^{\circ}, 120^{\circ}\right]$ cases when compared to all the other configruations. As observed from Fig. 9(c), in both configurations, the variation in $\dot{\varepsilon}$ (golden and green color) shows a similar trend as the $\beta=90^{\circ}$ case (cyan color) but the magnitude is higher near the end of the confluence regions $(x / h \sim 1.15)$ and from there onwards, the decay is much slower up to $x / h \approx 5$.

On the contrary, Figs. $8(\mathrm{~d})$ and $8(\mathrm{e})$ display a quite different behavior for the $\beta=\left[30^{\circ}, 150^{\circ}\right]$ pair. Even though both the configurations have the same confluence region $0 \leqslant x / h \leqslant 2$, there is a considerable variation in the deceleration and acceleration regions as seen from Fig. 9(c). For the $\beta=30^{\circ}$ case, the deceleration $(x / h \simeq 0)$ is higher leading to the lowest $U_{c \text {,min }}$ among all the configurations and also lower $U_{c, \text { max }}$ far downstream as seen in Figs. 9(a) and 9(b), whereas in the $\beta=150^{\circ}$ case, deceleration is slightly lower as compared to the $\beta=30^{\circ}$ case and $U_{c, \max }$ is also higher. This results in an asymmetry of $U_{c, \min }$ and $U_{c, \max }$ with respect to the $\beta=90^{\circ}$ case. As seen 

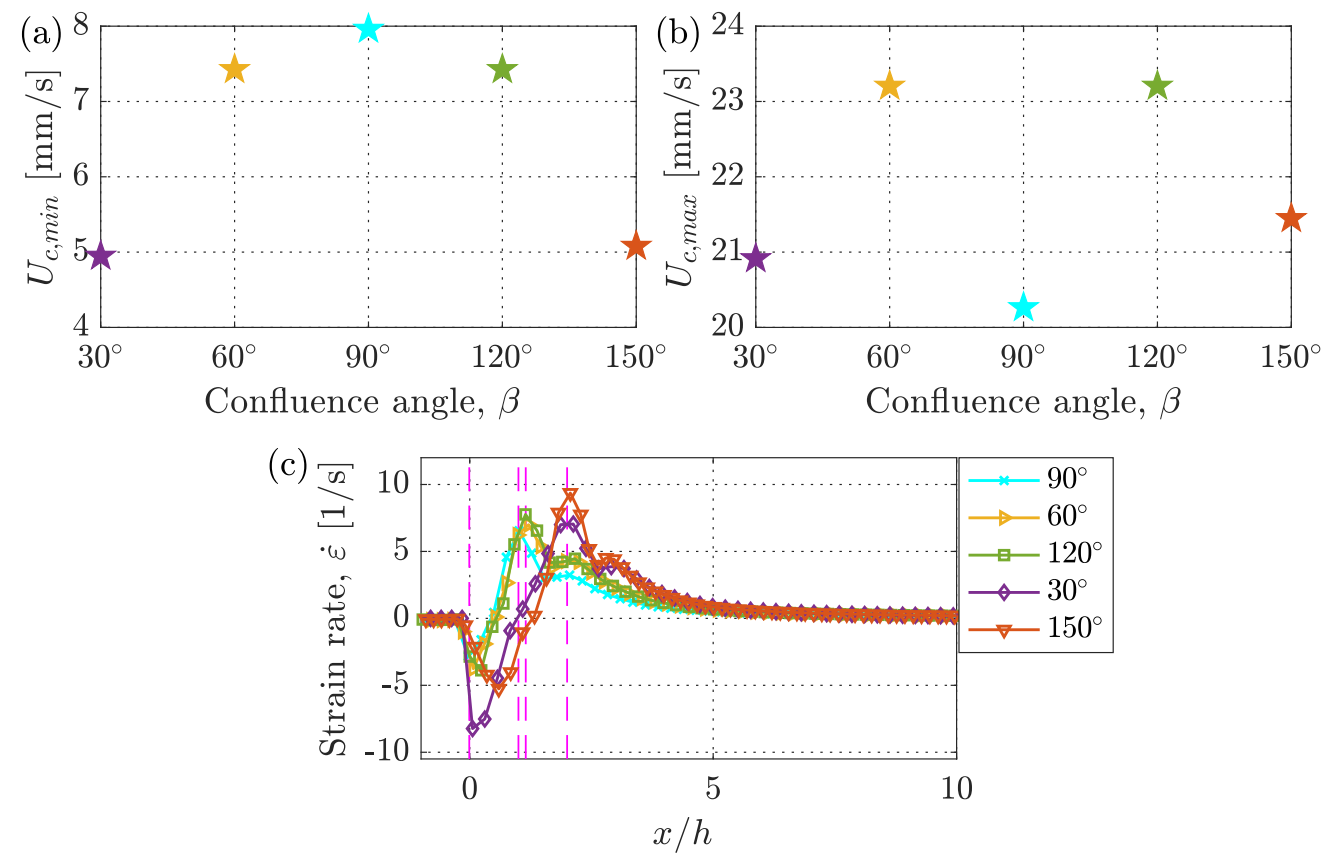

FIG. 9. Numerical plots of variation of minimum [panel (a)] and maximum velocity [panel (b)], and strain rate $\dot{\varepsilon}$ [panel (c)] along the centreline as a function of confluence angle $\beta$. The vertical dashed lines (magenta) in panel (c) denote the confluence regions for all the configurations. The confluence region starts at $x / h=0$ for all the configurations.

from Fig. 9(c) for $\beta=30^{\circ}$, the strain rate $\dot{\varepsilon}$ (purple color) is more steep than for the $\beta=150^{\circ}$ case leading to a lower peak value close to the end of confluence region $(x / h \sim 2)$. For $x / h>2$ onwards, the decay of $\dot{\varepsilon}$ in both cases follows a similar trend.

Thus, having affirmed the agreement between experimental measurements and numerical computations, we will now utilize purely the numerical computations for further analysis of confluence angle effects.

\section{REPLICATION OF CONFLUENCE ANGLE EFFECTS}

In this section, a strategic approach is attempted to comprehend the confluence angle effects. Two computations are performed utilizing the reference flow-focusing configuration $\left(\beta=90^{\circ}\right)$ with varying sheath flow inlet channel widths as illustrated in Fig. 10(a). Accordingly, the side channel width (SCW) of the "SCW-1.15h" configuration was set to $1.15 h$ matching the confluence region $(0 \leqslant x / h \leqslant 1.15)$ of the $\beta=\left[60^{\circ}, 120^{\circ}\right]$ configurations. Similarly, the "SCW- $2 h$ " configuration width was fixed to $2 h$ tallying with the confluence region $(0 \leqslant x / h \leqslant 2)$ of the $\beta=\left[30^{\circ}, 150^{\circ}\right]$ configurations. Other parameters such as flow rates of the core $\left(Q_{1}\right)$ and sheath $\left(Q_{2}\right)$ flows, rheologies of fluids are held fixed as described in the Secs. IIA1 and IIA2. The interfacial tension $\gamma=\Gamma_{e}=0.615 \mathrm{mN} \mathrm{m}^{-1}$, and the cross-sectional width $h$ of the central inlet and outlet channel were unchanged. This means that the sheath flow channel inlets are now rectangular in these cases.

Astonishingly, the flow in the SCW-1.15h configuration precisely replicates the morphological features of the wetted region and thread topologies of the $\beta=\left[60^{\circ}, 120^{\circ}\right]$ configurations as observed from Figs. 10(b) and 10(d). Once again this highlights the symmetry between these two cases. Whereas, the SCW- $2 h$ configuration captures and replicates the features of only the 


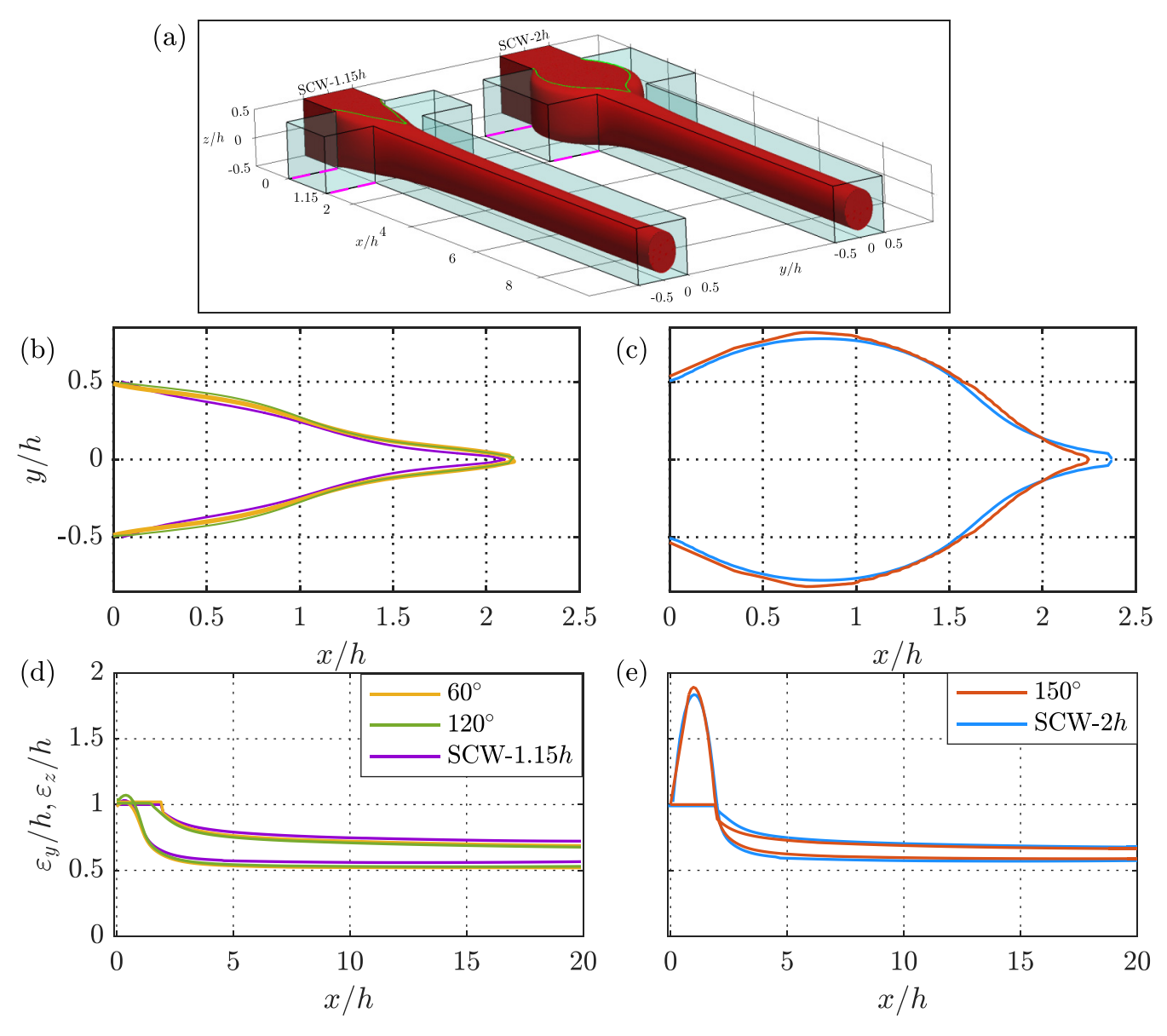

FIG. 10. (a) 3D view of the thread shapes for flow-focusing configurations with varying sheath flow channel widths (denoted by dashed magenta lines). Width of the side channel in the "SCW-1.5h" configuration corresponds to the confluence region $(0 \leqslant x / h \leqslant 1.15)$ of the $\beta=\left[60^{\circ}, 120^{\circ}\right]$ pair [see Figs. 3(b) and 3 (c)]. Likewise, width of the side channel in "SCW-2h" configuration corresponds to the confluence region $(0 \leqslant x / h \leqslant 2)$ of $\beta=\left[30^{\circ}, 150^{\circ}\right]$ pair [see Figs. 3(d) and 3(e)]. Panels (b) and (d) show the "SCW-1.5h" configuration wetted region, thread width $\left(\varepsilon_{y} / h\right)$ and height $\left(\varepsilon_{z} / h\right)$ plotted as a function of downstream positions $x / h$ overlapped with the wetted region and thread dimensions of $\beta=\left[60^{\circ}, 120^{\circ}\right]$ pair. Similar plots of panels (b) and (d) in panels (c) and (e) for the case of "SCW- $2 h$ " and $\beta=150^{\circ}$ configurations.

$\beta=150^{\circ}$ configuration as depicted in Figs. $10(\mathrm{c})$ and $10(\mathrm{e})$ (remember the asymmetry between the $\beta=\left[30^{\circ}, 150^{\circ}\right]$ configurations).

In the three cases, "SCW-1.15h" and $\beta=\left[60^{\circ}, 120^{\circ}\right]$, the sheath flow momentum normal to the core flow is attenuated by $13.4 \%$ in relative to the reference configuration $\left(\beta=90^{\circ}\right)$. For the "SCW$2 h^{\prime \prime}$ and $\beta=\left[30^{\circ}, 150^{\circ}\right]$ pair, the attenuation is around $50 \%$. Therefore, inclining the sheath flow channels at various confluence angles $\beta$ or setting the width of sheath flow channels of reference configuration to confluence region lengths, would essentially mean inducing the same magnitude of sheath flow momentum normal to the core flow. Thus, from these demonstrations, it is aptly clear that the magnitude of the sheath flow momentum at the confluence junction is the primary factor in controlling the morphological features of the wetted region and thread topologies except for near coflow $\left(\beta=30^{\circ}\right)$ case. 


\section{A COMMENT ON EFFECTIVE INTERFACIAL TENSION AND THREAD DETACHMENT}

The comprehensive systematic demonstrations utilizing geometrically varying flow-focusing setups in Sec. III A together with the alternative approach in previous Sec. IV indicates the significant role of $\Gamma_{e}$. As noticed, for a set flow rate and specified rheologies of fluids, the same value of interfacial tension modeling EIT $\left(\gamma=\Gamma_{e}=0.615 \mathrm{mN} \mathrm{m}^{-1}\right)$ deduced using the reference flow-focusing configuration $\left(\beta=90^{\circ}\right)$, very well reproduces the 3D flow characteristics for the respective geometrical configurations in the numerics. All the features such as the thread evolutions, morphologies of the wetted regions, and velocity fields showed good agreement with the experimental findings in Secs. III A and III C. In addition, the replication of 3D flow features of various confluence angle geometries captured via the modified geometries $\left(\beta=90^{\circ}\right)$, numerically with the same interfacial tension, further substantiate the robustness of our numerical procedure. Thus, as the Péclet number is large in the system, a sharp de facto interface exists in the experiments where EIT is expected. Since such a de facto interface can be associated with a near-immiscible behavior, utilizing an immiscible fluid solver, the above comprehensive set of results were captured and reproduced with ultralow values of interfacial tension $\gamma$. These evidences allows us to reassert that the weak interfacial tension $\gamma$ in the numerics models the ultra low but physically significant "effective" interfacial tension $\Gamma_{e}$ in experiments. Further, the ultralow value $\left(\gamma=\Gamma_{e}=0.615 \mathrm{mN} \mathrm{m}^{-1}\right)$ is of the same order of magnitude as the estimated $\Gamma_{e}\left(0.756 \mathrm{mN} \mathrm{m}^{-1}\right)$ in Sec. III B obtained utilizing the experimentally measured spatial thread shape $\left(\beta=90^{\circ}\right)$ and an effective capillary number dependent master curve. In fact, $\Gamma_{e}$ found in the present colloidal dispersion and solvent system is also in line and within the typical range $\left(10^{-4}-10^{1} \mathrm{mN} \mathrm{m}^{-1}\right)$ of experimentally observed EIT values involving complex fluids like colloidal or polymer dispersions and its own solvent [8].

An important and interesting aspect from the above illustrations is on the detachment of thread from the top and bottom channel walls. We find that, the above value of interfacial tension $\gamma$ in the numerical computations not only captured the wetted region shapes and wetted lengths $L_{w} / h$ accurately, but also steered the thread detachment from the channel walls. We also observed through a separate set of simulations that are not displayed here, that the wetted length $L_{w} / h \rightarrow \infty$ if $\gamma \rightarrow 0$, implying no detachment of thread from the top and bottom channel walls. Therefore, presence of ultralow interfacial tension $\gamma\left(\Gamma_{e}\right)$ is a decisive factor in order for the thread detachment to occur. Nevertheless, given the presence of high viscosity contrast $(\chi \simeq 4500)$ between the colloidal dispersion (core fluid) and its solvent (sheath fluid), Cubaud and Mason [30] predict that the process of thread detachment in miscible systems having large viscosity contrast [28,32], could occur naturally through the phenomenon of self-lubrication [36] depending on the fluid injection geometries and injection flow rates. According to this principle, the low-viscosity fluid enwraps the high-viscosity fluid to minimize the viscous dissipation of energy, leading to self-lubricated thread structures. However, as evident from the above observations, in high Péclet number microfluidic flows involving miscible systems with high-viscosity contrast, thread detachment is found to be clearly controlled by $\Gamma_{e}$ as well. Indeed, to have a finer understanding of the effects of EIT versus the ones associated with self-lubrication on the thread detachment, further detailed investigation is needed. For instance, variation of interfacial tension, viscosity contrast and flow rate ratios, and how these parameters affect the wetted length $L_{w} / h$ and thereby the thread detachment is worthwhile, and such investigations are beyond the scope of this present work.

\section{CONCLUSIONS}

The effective interfacial tension $\Gamma_{e}$ acting between the colloidal dispersion and its own solvent has been estimated utilizing the experimentally measured 3D spatial evolution of thread shape in the flow-focusing channel and an effective capillary number dependent master curve obtained from a simple scaling model [33]. We have verified from the 3D numerical computations, that the $\Gamma_{e}$ which minimizes the error between computed and experimental thread heights is close to the estimated $\Gamma_{e}$ utilizing the above method. The numerical computations were able to capture our experimental 
observations with good quantitative and qualitative agreement (both in terms of flow-topologies and flow-fields) for a range of confluence angles. By mapping the numerical observations with the experimental measurements, we gained insights into the influence of various physical mechanisms driving the process of thread detachment from the top and bottom walls in the channels. From these meticulous analyses, we find that in the case of high viscosity contrast miscible systems [28,32], the thread detachment occurring in the physical experiments of microchannels may not be entirely based on the phenomenon of self-lubrication [30] related with effect of viscous dissipation of energy, but also the effect of EIT induced by composition gradients plays a major role.

Moreover, these ultralow interfacial tensions in nonequilibrium miscible fluids are extremely difficult to evaluate in experimental measurements. The standard experimental techniques yielding reasonable results in the case of molecular miscible fluids are light scattering and spinning drop tensiometry [26]. However, these two techniques are observed to have drawbacks, in particular at the transition region due to mixing of the two fluids and difficulty to distinguish between the bulk fluids and interfacial region [26]. The recently explored new measurement strategies like examining the Saffman-Taylor instability in a Hele-Shaw cell $[7,8]$ with miscible complex fluids, and studying the dynamics of drop shape in spinning drop tensiometry [22] tested with miscible molecular systems are noteworthy developments. In addition to the above strategies, our method is also promising and could be a suitable choice.

From a technical perspective, varying the effect of confluence angle $\beta$ assists in the utilization of sheath flows to generate effective extensional flows. This, in turn, could facilitate to achieve optimal alignment of nanofibrils in colloidal dispersions [39,40]. A detailed investigation on the orientation and alignment of nanofibrils can be pursued, utilizing the velocity gradients of various flow configurations, including other relevant factors such as rotational diffusion, mobility, and rigidity of fibrils. Concluding, a thorough understanding of effects like EIT in miscible systems opens up possibilities in tuning, and controlling the material properties more efficiently via appropriate selection of microfluidic geometrical configuration based on the practical interest and application.

\section{ACKNOWLEDGMENTS}

Financial support by the Swedish Research Council for Environment, Agricultural Sciences and Spatial Planning (FORMAS) and Swedish Research Council (VR) are gratefully acknowledged. The simulations were performed on computer resources provided by the Swedish National Infrastructure for Computing (SNIC) at National Supercomputer Centre, Linköping, and at the Centre for High Performance Computing (PDC), KTH Royal Institute of Technology, Stockholm. Dr. Korneliya Gordeyeva is acknowledged for experimental assistance. The authors thank Dr. Christophe Brouzet for helpful discussions.

[1] D. D. Joseph, Fluid dynamics of two miscible liquids with diffusion and gradient stresses, Eur. J. Mech. B Fluids 9, 565 (1990).

[2] P. Garik, J. Hetrick, B. Orr, D. Barkey, and E. Ben-Jacob, Interfacial Cellular Mixing and a Conjecture on Global Deposit Morphology, Phys. Rev. Lett. 66, 1606 (1991).

[3] D. D. Joseph and Y. Y. Renardy, Fundamentals of Two-Fluid Dynamics. Part II: Lubricated Transport, Drops and Miscible Liquids (Springer, New York, 1993).

[4] D. M. Anderson, G. B. McFadden, and A. A. Wheeler, Diffuse-interface methods in fluid mechanics, Annu. Rev. Fluid Mech. 30, 139 (1998).

[5] J. Atencia and D. J. Beebe, Controlled microfluidic interfaces, Nature (London) 437, 648 (2005).

[6] D. J. Korteweg, Sur la forme que prennent les équations du mouvement des fluides si l'on tient compte des forces capillaires causées par des variations de densité, Arch. Neerl. Sci. Exactes Nat. 6, 1 (1901).

[7] D. Truzzolillo, S. Mora, C. Dupas, and L. Cipelletti, Off-Equilibrium Surface Tension in Colloidal Suspensions, Phys. Rev. Lett. 112, 128303 (2014). 
[8] D. Truzzolillo, S. Mora, C. Dupas, and L. Cipelletti, Nonequilibrium Interfacial Tension in Simple and Complex Fluids, Phys. Rev. X 6, 041057 (2016).

[9] P. G. Smith, T. G. Van De Ven, and S. G. Mason, Transient interfacial tension between two miscible fluids, J. Colloid Interface Sci. 80, 302 (1981).

[10] B. Zoltowski, Y. Chekanov, J. Masere, J. A. Pojman, and V. Volpert, Evidence for the existence of an effective interfacial tension between miscible fluids. 2. Dodecyl acrylate-poly (dodecyl acrylate) in a spinning drop tensiometer, Langmuir 23, 5522 (2007).

[11] R. X. Suzuki, F. W. Quah, T. Ban, M. Mishra, and Y. Nagatsu, Experimental study of miscible viscous fingering with different effective interfacial tension, AIP Adv. 10, 115219 (2020).

[12] S. Pramanik and M. Mishra, Linear stability analysis of Korteweg stresses effect on miscible viscous fingering in porous media, Phys. Fluids 25, 074104 (2013).

[13] S. Pramanik and M. Mishra, Nonlinear simulations of miscible viscous fingering with gradient stresses in porous media, Chem. Eng. Sci. 122, 523 (2015).

[14] S. Swernath, B. Malengier, and S. Pushpavanam, Effect of Korteweg stress on viscous fingering of solute plugs in a porous medium, Chem. Eng. Sci. 65, 2284 (2010).

[15] N. Bessonov, V. Volpert, J. A. Pojman, and B. Zoltowski, Numerical simulations of convection induced by Korteweg stresses in miscible polymer-monomer systems, Microgravity Sci. Technol. 17, 8 (2005).

[16] C. Chen, L. Wang, and E. Meiburg, Miscible droplets in a porous medium and the effects of Korteweg stresses, Phys. Fluids 13, 2447 (2001).

[17] C. Chen and E. Meiburg, Miscible displacements in capillary tubes: Influence of Korteweg stresses and divergence effects, Phys. Fluids 14, 2052 (2002).

[18] C.-Y. Chen, C.-W. Huang, H. Gadêlha, and J. A. Miranda, Radial viscous fingering in miscible Hele-Shaw flows: A numerical study, Phys. Rev. E 78, 016306 (2008).

[19] E. O. Dias and J. A. Miranda, Wavelength selection in Hele-Shaw flows: A maximum-amplitude criterion, Phys. Rev. E 88, 013016 (2013).

[20] E. Mossige, C. S.V., M. Islamov, S. Wheeler, and G. G. Fuller, Evaporation-induced Rayleigh-Taylor instabilities in polymer solutions, Philos. Trans. R. Soc. A 378, 20190533 (2020).

[21] J. A. Pojmanm, C. Whitmore, L. Turco, L. Maria, R. Lombardo, J. Marszalek, R. Parker, and B. Zoltowski, Evidence for the existence of an effective interfacial tension between miscible fluids: Isobutyric acid-water and 1-butanol- water in a spinning-drop tensiometer, Langmuir 22, 2569 (2006).

[22] A. Carbonaro, L. Cipelletti, and D. Truzzolillo, Ultralow effective interfacial tension between miscible molecular fluids, Phys. Rev. Fluids 5, 074001 (2020).

[23] V. Shevtsova, Y. Gaponenko, V. Yasnou, A. Mialdun, and A. Nepomnyashchy, Two-scale wave patterns on a periodically excited miscible liquid-liquid interface, J. Fluid. Mech. 795, 409 (2016).

[24] S. E. May and J. V. Maher, Capillary-Wave Relaxation for a Meniscus Between Miscible Liquids, Phys. Rev. Lett. 67, 2013 (1991).

[25] P. Cicuta, A. Vailati, and M. Giglio, Capillary-to-bulk crossover of nonequilibrium fluctuations in the free diffusion of a near-critical binary liquid mixture, Appl. Opt. 40, 4140 (2001).

[26] D. Truzzolillo and L. Cipelletti, Off-equilibrium surface tension in miscible fluids, Soft Matter 13, 13 (2017).

[27] T. Cubaud and T. G. Mason, Folding of Viscous Threads in Diverging Microchannels, Phys. Rev. Lett. 96, 114501 (2006).

[28] T. Cubaud and S. Notaro, Regimes of miscible fluid thread formation in microfluidic focusing sections, Phys. Fluids 26, 122005 (2014).

[29] T. Cubaud, Swelling of Diffusive Fluid Threads in Microchannels, Phys. Rev. Lett. 125, 174502 (2020).

[30] T. Cubaud and T. Mason, Interacting viscous instabilities in microfluidic systems, Soft Matter 8, 10573 (2012).

[31] O. Bonhomme, J. Leng, and A. Colin, Microfluidic wet-spinning of alginate microfibers: A theoretical analysis of fiber formation, Soft Matter 8, 10641 (2012).

[32] T. Cubaud and T. G. Mason, High-viscosity fluid threads in weakly diffusive microfluidic systems, New J. Phys. 11, 075029 (2009). 
[33] K. Gowda, C. Brouzet, T. Lefranc, L. Söderberg, and F. Lundell, Effective interfacial tension in flowfocusing of colloidal dispersions: 3D numerical simulations and experiments, J. Fluid. Mech. 876, 1052 (2019).

[34] C. W. Hirt and B. D. Nichols, Volume of fluid (VoF) method for the dynamics of free boundaries, J. Comput. Phys. 39, 201 (1981).

[35] E. OpenCFD, OpenFOAM v1806 Userguide (ESI-OpenCFD, 2018).

[36] D. Joseph, K. Nguyen, and G. Beavers, Nonuniqueness and stability of the configuration of flow of immiscible fluids with different viscosities, J. Fluid. Mech. 141, 319 (1984).

[37] K. M. O. Håkansson, A. B. Fall, F. Lundell, S. Yu, C. Krywka, S. V. Roth, G. Santoro, M. Kvick, L. Prahl-Wittberg, L. Wågberg, and L. D. Söderberg, Hydrodynamic alignment and assembly of nanofibrils resulting in strong cellulose filaments, Nat. Commun. 5, 4018 (2014).

[38] N. Mittal, F. Ansari, K. Gowda.V, C. Brouzet, P. Chen, P. T. Larsson, S. V. Roth, F. Lundell, L. Wagberg, N. A. Kotov, and L. D. Söderberg, Multiscale control of nanocellulose assembly: Transferring remarkable nanoscale fibril mechanics to macroscale fibers, ACS Nano 12, 6378 (2018).

[39] C. Brouzet, N. Mittal, L. D. Söderberg, and F. Lundell, Size-dependent orientational dynamics of Brownian nanorods, ACS Macro Lett. 7, 1022 (2018).

[40] C. Brouzet, N. Mittal, F. Lundell, and L. D. Söderberg, Characterizing the orientational and network dynamics of polydisperse nanofibers on the nanoscale, Macromolecules 52, 2286 (2019).

[41] D. Truzzolillo, V. Roger, C. Dupas, S. Mora, and L. Cipelletti, Bulk and interfacial stresses in suspensions of soft and hard colloids, J. Phys. Condens. Matter 27, 194103 (2015).

[42] T. Saito, S. Kimura, Y. Nishiyama, and A. Isogai, Cellulose nanofibers prepared by Tempo-mediated oxidation of native cellulose, Biomacromolecules 8, 2485 (2007).

[43] A. Isogai, T. Saito, and H. Fukuzumi, Tempo-oxidized cellulose nanofibers, Nanoscale 3, 71 (2011).

[44] F. Martoïa, P. J. J. Dumont, L. Orgéas, M. N. Belgacem, and J. L. Putaux, Micro-mechanics of electrostatically stabilized suspensions of cellulose nanofibrils under steady state shear flow, Soft Matter 12, 1721 (2016).

[45] O. Nechyporchuk, M. N. Belgacem, and F. Pignon, Current progress in rheology of cellulose nanofibril suspensions, Biomacromolecules 17, 2311 (2016).

[46] L. Geng, N. Mittal, C. Zhan, F. Ansari, P. R. Sharma, X. Peng, B. S. Hsiao, and L. Söderberg, Understanding the mechanistic behavior of highly charged cellulose nanofibers in aqueous systems, Macromolecules 51, 1498 (2018).

[47] L. H. Switzer and D. J. Klingenberg, Simulations of fiber floc dispersion in linear flow fields, Nordic Pulp Pap. Res. J. 18, 141 (2003).

[48] M. Doi and S. F. Edwards, The Theory of Polymer Dynamics, Vol. 73 (Oxford University Press, Oxford, UK, 1988).

[49] G. Marrucci and N. Grizzuti, The effect of polydispersity on rotational diffusivity and shear viscosity of rodlike polymers in concentrated solutions, J. Polym. Sci. Polym. Lett. Ed. 21, 83 (1983).

[50] G. Marrucci and N. Grizzuti, Predicted effect of polydispersity on rodlike polymer behaviour in concentrated solutions, J. Non-Newtonian Fluid Mech. 14, 103 (1984).

[51] A. W. Chow, G. G. Fuller, D. G. Wallace, and J. A. Madri, Rheo-optical response of rodlike, shortened collagen protein to transient shear flow, Macromolecules 18, 805 (1985).

[52] R. Pecora, Dynamics of rodlike macromolecules in semidilute solutions, J. Polym Sci.: Polym. Symp. 73, 83 (1985).

[53] S. S. Rogers, P. Venema, L. M. C. Sagis, E. van der Linden, and A. M. Donald, Measuring the length distribution of a fibril system: A flow birefringence technique applied to amyloid fibrils, Macromolecules 38, 2948 (2005).

[54] T. Rosén, B. S. Hsiao, and L. D. Söderberg, Elucidating the opportunities and challenges for nanocellulose spinning, Adv. Mater. 33, 2001238 (2020).

[55] D. Huang, E. Swanson, C. Lin, J. Schuman, W. Stinson, W. Chang, M. Hee, T. Flotte, K. Gregory, and C. A. Puliafito, Optical coherence tomography, Science 254, 1178 (1991).

[56] W. Drexler and J. G. Fujimoto, Optical Coherence Tomography: Technology and Applications (Springer Science \& Business Media, Berlin, 2008). 
[57] R. A. Leitgeb, R. M. Werkmeister, C. Blatter, and L. Schmetterer, Doppler optical coherence tomography, Prog. Retinal Eye Res. 41, 26 (2014).

[58] J. Roenby, H. Bredmose, and H. Jasak, A computational method for sharp interface advection, R. Soc. Open Sci. 3, 160405 (2016).

[59] J. U. Brackbill, D. B. Kothe, and C. Zemach, A continuum method for modeling surface tension, J. Comput. Phys. 100, 335 (1992).

[60] T. Cubaud and T. G. Mason, Capillary threads and viscous droplets in square microchannels, Phys. Fluids 20, 053302 (2008).

[61] G. B. Jeffery, The motion of ellipsoidal particles immersed in a viscous fluid, Proc. R. Soc. London, Ser. A 102, 161 (1922).

[62] K. M. O. Håkansson, F. Lundell, L. Prahl-Wittberg, and L. D. Söderberg, Nanofibril alignment in flow focusing: Measurements and calculations, J. Phys. Chem. B 120, 6674 (2016). 